\title{
Layered Estimation of Atmospheric Mesoscale Dynamics From Satellite Imagery
}

\author{
Patrick Héas, Etienne Mémin, Nicolas Papadakis, and André Szantai
}

\begin{abstract}
In this paper, we address the problem of estimating mesoscale dynamics of atmospheric layers from satellite image sequences. Due to the great deal of spatial and temporal distortions of cloud patterns and because of the sparse 3-D nature of cloud observations, standard dense-motion field-estimation techniques used in computer vision are not well adapted to satellite images. Relying on a physically sound vertical decomposition of the atmosphere into layers, we propose a dense-motion estimator dedicated to the extraction of multilayer horizontal wind fields. This estimator is expressed as the minimization of a global function including data and spatio-temporal smoothness terms. A robust data term relying on the integrated-continuity equation massconservation model is proposed to fit sparse-transmittance observations related to each layer. A novel spatio-temporal smoother derived from large eddy prediction of a shallow-water momentumconservation model is used to build constraints for large-scale temporal coherence. These constraints are combined in a global smoothing framework with a robust second-order smoother, preserving divergent and vorticity structures of the flow. For optimization, a two-stage motion estimation scheme is proposed to overcome multiresolution limitations when capturing the dynamics of mesoscale structures. This alternative approach relies on the combination of correlation and optical-flow observations in a variational context. An exhaustive evaluation of the novel method is first performed on a scalar image sequence generated by direct numerical simulation of a turbulent 2-D flow. By qualitative comparisons, the method is then assessed on a METEOSAT image sequence.
\end{abstract}

Index Terms-Atmospheric-motion estimation, correlationbased vector interpolation, filtered shallow-water equations, integrated continuity equation (ICE), layer transmittance, optical flow, spatio-temporal smoothing, variational methods.

\section{INTRODUCTION}

$\mathbf{T}$ HE ANALYSIS of complex fluid-flow behaviors is a major scientific issue. In particular, understanding atmospheric dynamics is of great importance for meteorologists interested in weather forecasting, climate prediction, singularsystem analysis, etc. Surface stations, balloons (including radiosonde), and, more recently, in-flight aircraft measurements and satellite images characterized by low spatial and temporal resolutions have improved the estimation of wind fields and have been an important step for a better understanding of me-

Manuscript received October 13, 2006; revised March 27, 2007

P. Héas, E. Mémin, and N. Papadakis are with the Institut National de Recherche en Informatique et en Automatique/Institut de recherche en informatique et systmes aléatoires, Campus de Beaulieu, 35042 Rennes Cedex, France (e-mail: Patrick.Heas@irisa.fr; Etienne.Memin@irisa.fr; Nicolas.Papadakis@irisa.fr).

A. Szantai is with the Laboratoire de Météorologie Dynamique, Ecole Polytechnique, 91128 Palaiseau, France (e-mail: Andre.Szantai@lmd. polytechnique.fr).

Digital Object Identifier 10.1109/TGRS.2007.906156 teorological phenomena. However, the measurements provided by the network's temporal and spatial resolutions of in situ measurements may be insufficient for the analysis of mesoscale dynamics. Let us recall that mesoscale dynamics is generally defined to include the study of motion systems that have horizontal scales in the range of about $10-1000 \mathrm{~km}$. It includes circulation ranging from thunderstorms and internal gravity waves at the small end of the scale to fronts and hurricanes at the large end [17]. In particular, meteorological in situ data are excessively sparse over the southern hemisphere. Such a lack of data makes the establishment of accurate numerical prediction difficult. Recently, in an effort to avoid these limitations, an increased interest has been devoted to motion extraction from image sequences from a new generation of geostationary satellites, characterized by finer spatial and temporal resolutions.

The analysis of motion in such sequences is particularly challenging due to the great deal of spatial and temporal distortions that luminance patterns exhibit in imaged atmospheric phenomena. Standard techniques from computer vision, originally designed for rigid motions and stable salient features along time, are not well adapted in this context. Winds have been derived from the motion of clouds on satellite images for about three decades [21], [34] and are used operationally in forecast models. However, only few constraints, based mainly on quality and consistency of the extracted motion vectors, are applied, and the estimated wind fields have a limited spatial coverage. Recently, computer vision methods have been adapted for fluiddedicated dense estimation in order to characterize atmospheric motion [7], [8], [26].

Nevertheless, we will show that, due to the underlying 3-D nature of the scene, the employed dynamical models remain unadapted to satellite observations. Furthermore, such methods may fail to accurately characterize motion associated with mesoscale structures. Thus, the design of an appropriate approach modeling the physics of 3-D atmosphere dynamics constitutes a wide-open domain of research. This paper is a contribution in this direction.

Rather than coupling the motion-vector-estimation process to a complex and complete numerical meteorological circulation model, we propose to incorporate "some" dynamics in the motion estimation scheme in the form of an adaptation of Navier-Stokes equations to satellite imagery. The objective being in line with the 3-D reconstruction of atmospheric horizontal winds. Alternatively, the challenge also consists in providing accurate estimators that is able to tackle the motion complexity of sparse and noisy structures.

In this paper, we propose original methods responding to this problem. The remainder of this paper is organized as follows. 
In the following section, in order to motivate our approach, an overview on existing optical-flow-estimation methods is presented. Then, in Section III, a mass-conservation model for an atmosphere decomposed into a stack of layers is introduced. This model constitutes the physical background of the multilayer dense estimator, which is then described. In the perspective of adapting motion analysis to mesoscale, we propose in Section IV a two-stage decomposition estimation scheme. We propose to combine the ability of correlational and variational approaches and to enhance spatio-temporal consistency by using a simplified shallow-water model. Finally, results on METEOSAT image sequences presented in Section V demonstrate the interest of our approach.

\section{RELATED WORK ON OPTICAL-FLOW ESTIMATION}

The problem of wind-field estimation in an image sequence $I(x, y, t)$ consists in characterizing the real 3-D atmospheric motion from observations in the projected image plane. This problem is a complex one, for which we have only access to projected information on cloud positions and spectral signatures provided by satellite-observation channels.

\section{A. Real Projected Wind Fields and Optical Flow}

Spatial horizontal coordinates $(x, y)$ are denoted by s. To avoid tackling the 3 -D wind-field $\mathbf{V}(\mathbf{s}, z, t)$ reconstruction problem, up to now, all the developed wind-field-estimation methods rely on the assumption of unexistent vertical winds and estimate of an average horizontal wind.

The apparent motion $\mathbf{v}$, perceived through image-intensity variations, is called optical flow in the computer vision community. All optical-flow-estimation methods rely on the temporal conservation of some invariants. Although geometric invariants may be relevant for matching across frames (e.g., point, contour tracking) [13], they can only be extracted in sufficiently structured areas and, thus, lead to sparse-motion measurements. The most common invariants used are photometric ones which can easily be extracted and lead to dense measurements. In particular, for satellite images, atmospheric motion is revealed by a function $F($. of the intensity of clouds, which are assumed to be passive tracers. From these considerations, two schemes can be derived for motion estimation: a nonlinear formulation matching consecutive images and a linear differential formulation. The first formulation leads to a nonlinear system of equations valid for every kind of motion, whereas the second holds only for small displacements and is often illconditioned.

1) Brightness-Constancy Model: For the special case of gray-level constancy assumption, $F($.$) is the identity function,$ and apparent motion $\mathbf{v}(\mathbf{s}, t)$ is equal to an average horizontal wind. This assumption leads for the nonlinear formulation to the displaced-frame-difference (DFD) equation

$$
I(\mathbf{s}+\mathbf{v}, t+1)=I(\mathbf{s}, t)
$$

while, for the differential formulation, one obtains the wellknown optical-flow constraint (OFC) equation

$$
\frac{d I}{d t}=\mathbf{v} \cdot \nabla I(\mathbf{s}, t)+I_{t}(\mathbf{s}, t)=0
$$

An important remark is that, for image sequences showing evolving atmospheric phenomena, the brightness-consistency assumption does not allow us to model temporal distortions of luminance patterns caused by 3-D flow transportation.

2) Integrated Continuity Equation (ICE): For atmospheric image sequences, the underlying physical laws determining the atmospheric evolution constitute an inestimable source of a priori information to design an appropriate data model. Interesting models may be derived from the 3-D mass-conservation law, the so-called continuity equation

$$
\frac{1}{\rho} \frac{d \rho}{d t}+\nabla \cdot \mathbf{V}=0
$$

where $\rho$ denotes a 3-D density function. Let us remark that a $3-\mathrm{D}$ wind field $\mathbf{V}$ is considered in the previous equation. Image intensity may be linked to the vertical integration of (3) [11]. Unlike the brightness-consistency assumption, such models can compensate mass departures observed in the image plane by associating 2-D divergence to brightness variations. In this case, we redefine apparent motion $\mathbf{v}$ as a density-weighted average of the original 3-D horizontal velocity field $\mathbf{v}_{\text {real }}$

$$
\mathbf{v}=\frac{\int \rho \mathbf{v}_{\text {real }} d z}{\int \rho d z}
$$

Neglecting mass exchanges via vertical motion on planar integral boundaries, in [11] and [2], the authors demonstrated that the integration of (3) leads to a mass-conservation model called ICE

$$
\left(\int \rho d z\right)_{t}+\mathbf{v} \cdot \nabla\left(\int \rho d z\right)+\nabla \cdot \mathbf{v}\left(\int \rho d z\right)=0 .
$$

In the case of transmittance imagery, $I=\int \rho d z$ and (5) can directly be used [11] assuming no vertical motion on the planar integral boundaries. The image formation model for satellite infrared imagery is slightly different. In [7], the authors have directly assumed the unrealistic hypothesis that infrared pixel values $I$ were proportional to density integrals: $I \propto \int \rho d z$. This assumption has enabled them to rewrite (5) as

$$
I_{t}+\mathbf{v} \cdot \nabla I+I \operatorname{div}(\mathbf{v})=0
$$

In [43], the authors proposed an inversely proportional approximation for infrared measurements: $I \propto\left(\int \rho d z\right)^{-1}$. Such an assumption yields to the same equation except for a minus sign appearing in front of the divergence term. By time integration of (6), an equivalent nonlinear DFD formulation [(1)] can also be recovered [7]

$$
I(\mathbf{s}+\mathbf{v}, t+1) \exp \operatorname{div}(\mathbf{v})-I(\mathbf{s}, t)=0 .
$$

These two models constitute adaptations of the ICE model for the case of satellite infrared imagery. Experimentally, these 


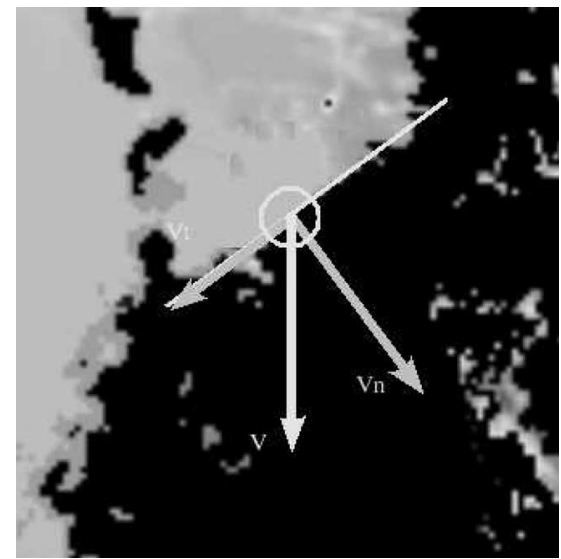

Fig. 1. Aperture problem for the estimation of atmospheric motion $\mathbf{v}$ in areas of low local curvatures of the image isointensity curves. Locally (circle), only the velocity $\mathbf{v}_{n}$ normal to the tangent of the isocurve can be estimated while the tangential component $\mathbf{v}_{t}$ will remain undetermined.

approaches have proved to be relevant in comparison to models based on the OFC [(2)] or the DFD [(1)]. However, from a physical point of view, they rely on nonrealistic approximations. We will see in Section III-A how to reinforce the theoretical background of such an approach by adapting image observations.

The formulations of (1), (2), (6), and (7) cannot be used alone, as they provide only one equation for two unknowns at each spatio-temporal locations $(\mathbf{s}, t)$ with, therefore, a 1-D family of solutions in general. In the case of (2), this is the well-known aperture problem where the so-called normal flow is estimated while the tangential-velocity component remains undetermined. The aperture problem is illustrated in Fig. 1. In order to remove this ambiguity and make the estimation robust, one must rely on other assumptions. The most common assumptions consist in improving the spatial coherence of windfield estimates. Such assumptions are expressed by prior models on spatio-temporal dependence. This allows the propagation of information in uniform regions and reduction of sensitivity to noise. The choice of a particular smoothness function characterizes a fundamental difference between large families of opticalflow techniques. Among all the smoothing schemes, one can roughly distinguish disjoint from global approaches.

\section{B. Disjoint Spatial-Smoothing Schemes}

In these approaches, one considers neighborhoods $W(\mathbf{s})$ centered at pixel locations. An independent parametric field is locally estimated on each of these supports. The most common types of parametric representations are the constant, affine, and quadratic flow fields.

A first category of disjoint local smoothing methods relies on the OFC [(2)]. Motion is estimated using a standard linear least squares approach

$$
\widehat{\mathbf{v}}=\arg \min _{u} \int_{W(\mathbf{s})} h(\mathbf{s}-\mathbf{y})\left(\mathbf{u} \cdot \nabla I(\mathbf{y})+I_{t}(\mathbf{y})\right)^{2} d \mathbf{y}
$$

where $h(\mathbf{s})$ is a weighting function, such as a spatial Gaussian kernel for example, which controls the influence of a given location in the neighborhood $W(\mathbf{s})$ of the estimation support. If the motion is assumed to be locally constant, this estimator corresponds essentially to the work of Lucas and Kanade [22]. These methods present the drawback to be sensitive to noise and too often lead to ill-conditioned problems in the case of weak-intensity gradients.

In meteorology, classical approaches are Euclidean correlation-based matchings, which corresponds to the DFD constraint (1) associated with a locally constant field and a $\boldsymbol{L}^{2}$ norm [1], [10], [21], [30], [34], [37]. Using a discrete state space of vectors $\{\mathbf{u}\}$, discrete correlation approaches consist of estimating a displacement vector $\mathbf{v}$ at point $\mathbf{s}$ as

$$
\mathbf{v}(\mathbf{s})=\arg \min _{\mathbf{u}} \sum_{\mathbf{r} \in W(\mathbf{s})} \mathcal{C}(I(\mathbf{r}+\mathbf{u}, t+1), I(\mathbf{s}, t))
$$

where $\mathcal{C}($.$) denotes a dissimilarity function. On the one hand,$ they constitute fast methods, generally locally robust to noise, and which are able to estimate large displacement of fine structures. On the other hand, these approaches suffer from several deficiencies: traceable cloud features must be sufficiently contrasted and must persist over time on consecutive images. Thus, the estimation is prone to erroneous spatial variability. Furthermore, any disjoint technique is based on independent local estimations of cloudy regions which results in the estimation of sparse and possibly spatially incoherent vector fields. We will see in Section IV-B how to take advantage of these approaches while avoiding erroneous solutions.

\section{Global Spatial-Smoothing Schemes}

Global smoothing schemes can be used to overcome the previous limitations. These methods model spatio-temporal dependencies on the complete image domain denoted by $\Omega$. Thus, dense velocity fields are estimated even in the case of noisy and low-contrasted observations. Functional models form the vast majority of the dense-estimation methods. Their popularity is due to their flexibility. Indeed, these methods enable in a common framework to handle discontinuities or occlusion zones and to design dedicated smoothing functions to particular phenomena. In addition to constraints linking unknown velocities to image observations, these approaches introduce explicitly a priori smoothing functions, which enable to solve the underdetermined problem. More precisely, the motionestimation problem is defined as the global minimization of an energy function composed of two components

$$
J(\mathbf{v}, I)=J_{d}(\mathbf{v}, I)+\alpha J_{r}(\mathbf{v}) .
$$

The first component $J_{d}(\mathbf{v}, I)$, called the data term, expresses the constraint linking unknowns to observations while the second component $J_{r}(\mathbf{v})$, called the smoothing term, enforces the solution to follow some smoothness properties. In the previous expression, $\alpha>0$ denotes a parameter controlling the balance between the smoothness and the global adequacy to the observation model. In this framework, 
Horn and Schunck [18] derived the following data term from the OFC [(2)]:

$$
J_{d}(\mathbf{v}, I)=\int_{\Omega}\left(I_{t}(\mathbf{s})+\mathbf{v}(\mathbf{s}) \cdot \nabla I(\mathbf{s})\right)^{2} d \mathbf{s}
$$

and combined it to a first-order smoothing term

$$
J_{r}(\mathbf{v})=\int_{\Omega} \|\left(\nabla u(\mathbf{s})\left\|^{2}+\right\| \nabla v(\mathbf{s}) \|^{2}\right) d \mathbf{s}
$$

where $u$ and $v$ are the two spatial components of a velocity field $\mathbf{v}[20]$. In the case of fluid flows, using the previously defined ICE model [(6)] leads to the functional

$$
J_{d}(\mathbf{v}, I)=\int_{\Omega}\left(I_{t}(\mathbf{s})+\mathbf{v}(\mathbf{s}) \cdot \nabla I(\mathbf{s})+I(\mathbf{s}) \operatorname{div}(\mathbf{v}(\mathbf{s}))\right)^{2} d \mathbf{s} .
$$

Moreover, it can be demonstrated that a first-order smoothing is not adapted as it favors the estimation of velocity fields with low divergence and low vorticity. A second-order smoothing of the vorticity and the divergence of the apparent motion field can advantageously be considered as proposed in [36]

$$
J_{r}(\mathbf{v})=\int_{\Omega}\left(\|\nabla \operatorname{curl} \mathbf{v}(\mathbf{s})\|^{2}+\|\nabla \operatorname{divv}(\mathbf{s})\|^{2}\right) d \mathbf{s} .
$$

To circumvent the difficulty of implementing the second-order smoothness constraint, this smoothing term can be simplified, from a computational point of view, to two interleaved firstorder div-curl smoothings based on auxiliary variables $\zeta_{\mathbf{v}}$ and $D_{\mathrm{v}}$ approximating the vorticity and the divergence of the flow [7]. Thus, we have

$$
\begin{aligned}
J_{r}(\mathbf{v})=\int_{\Omega} & \left(\operatorname{curl}(\mathbf{v}(\mathbf{s}))-\zeta_{\mathbf{v}}(\mathbf{s})\right)^{2}+\mu\left\|\nabla \zeta_{\mathbf{v}}(\mathbf{s})\right\|^{2} \\
& +\left(\operatorname{div}(\mathbf{v}(\mathbf{s}))-D_{\mathbf{v}}(\mathbf{s})\right)^{2}+\mu\left\|\boldsymbol{\nabla} D_{\mathbf{v}}(\mathbf{s})\right\|^{2} d \mathbf{s}
\end{aligned}
$$

where $\mu$ is a positive smoothing parameter. Another way to implement such high-order smoothing has been proposed recently and relies on mimetic finite differences [42]. In both approaches, a common way to deal with the boundedness of the spatial domain $\Omega$ is to use Neumann boundary conditions. They specify that, on image edges, velocity normal derivatives are equal to zero.

A large linear system is obtained by discretizing the previous functions in a finite-difference scheme. We obtain in this case a Bayesian maximum a posteriori estimation approach, where the velocity field is modeled by a Markov random field [15]. The related energy is a quadratic function which can be minimized by alternatively solving large systems for unknowns $\mathbf{v}, \zeta_{\mathbf{v}}$, and $D_{\mathbf{v}}$ in an iterative scheme using, for instance, Gauss-Seidel method. For better and faster convergence, an incremental coarse-to-fine multigrid technique can be used [5], [25]. The approach proposed in [25] consists of a search for an incremental solution, where the unknown increment $\delta \mathbf{v}(\mathbf{s})=P(\theta(\mathbf{s}))$ is defined on a subspace $\theta(\mathbf{s})$ of solutions via a parametric model $P$. Multigrid estimation is performed in an incremental scheme, which implies to adapt the previously defined functions. Such an adaptation will be detailed as follows for the multiresolution scheme. However, one should not confuse these two techniques which operate in a slightly different way.

In order to preserve discontinuities and to handle occlusion zones, which are frequent in satellite imagery due to the stratified and 3-D nature of the observed clouds, robust estimation constitutes an interesting approach [4], [29], [39]. In the case of functional minimization, a robust penalty function $\phi_{d}$ may be introduced in the data term in attenuating the effect of observations deviating significantly from the model [4]. Similarly, a robust penalty function $\phi_{r}$ can be used if one wants to handle implicitly the spatial discontinuities of the vorticity and divergence maps. M-estimator penalty functions can be chosen for their advantageous minimization properties [4], [14], [16]. In particular, Leclerc and Geman-Mc Clure penality functions proved to be suitable, both for the observation model and for the smoother.

In the image plane, these discontinuities are nevertheless difficult to relate to abrupt variations of cloud heights. Moreover, clouds belonging to a layer form unconnected regions, which should interact during the motion-estimation process. To overcome these limitations, we will propose in Section III-B a layer-dedicated motion estimator, providing dense-motion fields related to each layer.

One major problem with optical-flow differential formulation is the estimation of large displacements. OFC [(2)] or ICE [(6)] models rely on the assumption that the image-intensity function can be locally efficiently approximated by a linear function. Since the larger the displacement the more narrow the linearity domain, large displacements are difficult to estimate directly. The multiresolution approach is a common way to overcome this limitation by creating an image pyramid, constructed by successive low-pass filtering and down sampling of the original images. Within such a framework, main components $\tilde{\mathbf{v}}$ of displacements $\mathbf{v}$ are first estimated at a coarse resolution, where motion amplitudes should be sufficiently reduced in order to make the differential-formulation valid. Then, the estimation is progressively refined while going down in the pyramid levels [3], [4], [25], [39]. This approach is, to some extent, similar to the multigrid scheme introduced previously, the main difference being that multigrid approaches are based on a unique representation of the data while a multiresolution scheme uses a data pyramidal structure. In both cases, the displacement component estimated previously is used to derive a motion-compensated linear differential formulation, which enables the estimation of a displacement increment $\mathbf{v}^{\prime}$. Noting that $\mathbf{v}=\tilde{\mathbf{v}}+\mathbf{v}^{\prime}$ for the integrated ICE [(7)], this is obtained by a first-order Taylor expansion of $I(\mathbf{s}+\mathbf{v}(\mathbf{s}), t+1) \exp (\operatorname{div}(\mathbf{v}(\mathbf{s})))$ around the point $\mathbf{s}+\tilde{\mathbf{v}}(\mathbf{s})$. Thus, denoting by $\tilde{I}(\mathbf{s})$ the motion-compensated image $I(\mathbf{s}+\tilde{\mathbf{v}}, t+1)$ and omitting time coordinates, the 
linearization yields a new robust data term $J_{d}(\mathbf{v}, I)$, which is defined as

$$
\begin{array}{r}
\int_{\Omega} \phi_{d}\left\{\operatorname { e x p } \operatorname { d i v } \tilde { \mathbf { v } } ( \mathbf { s } ) \left([\tilde{I}(\mathbf{s}) \boldsymbol{\nabla} \operatorname{div}(\tilde{\mathbf{v}}(\mathbf{s}))+\nabla \tilde{I}(\mathbf{s})]^{\top} \mathbf{v}^{\prime}(\mathbf{s})\right.\right. \\
+\tilde{I}(\mathbf{s}))-I(\mathbf{s})\} d \mathbf{s}
\end{array}
$$

and (15) is changed to a new robust div-curl smoothing term

$$
\begin{aligned}
J_{r}(\mathbf{v})= & \int_{\Omega}\left(\operatorname{div}\left(\tilde{\mathbf{v}}(\mathbf{s})+\mathbf{v}^{\prime}(\mathbf{s})\right)-D_{\mathbf{v}}(\mathbf{s})\right)^{2}+\mu \phi_{r}\left(\left\|\boldsymbol{\nabla} D_{\mathbf{v}}(\mathbf{s})\right\|\right) \\
& +\left(\operatorname{curl}\left(\tilde{\mathbf{v}}(\mathbf{s})+\mathbf{v}^{\prime}(\mathbf{s})\right)-\zeta_{\mathbf{v}}(\mathbf{s})\right)^{2}+\mu \phi_{r}\left(\left\|\boldsymbol{\nabla} \zeta_{\mathbf{v}}(\mathbf{s})\right\|\right) d \mathbf{s} .
\end{aligned}
$$

At a given resolution level, the main component $\tilde{\mathbf{v}}$ obtained at a coarser level is refined with the increment $\mathbf{v}^{\prime}$. Therefore, the large displacement problem is tackled by decomposition of the nonlinear estimation into successive linear estimations. However, since the multiresolution scheme estimates the maincomponent displacements only at coarse resolutions where small photometric structures are rubbed out, this approach enables the characterization of large displacements of small structures only in the case when their individual motions are close enough to the main component's one. This is often not the case for a layered atmosphere. For instance, mesoscale structures such as cirrus filaments may be characterized by large displacements which appear to be completely different from the motion of the layers underneath. Another disadvantage characterizing the method is the additional noise due to the calculation of motion-compensated images for each resolution by the use of interpolation methods. An alternative estimation scheme for large displacements will be proposed in Section IV-A.

\section{Dense-Motion Estimator DedicAted TO ATMOSPHERIC LAYERS}

Based on a previous work on dense estimation for 2-D fluid flows, an original motion estimator acting at the lower range of mesoscale is proposed to cope with the 3-D nature of atmospheric flows.

\section{A. Dynamical Observation Model for Layers}

As it has been aforementioned, the ICE model (6) relies on strong assumptions in the case of satellite infrared imagery. However, it has been demonstrated that the ICE model is well suited for an image sequence of transmittance measurements. In this section, we propose a physically sound approximation in computing for transmittance images related to atmospheric layers using pressure images of top of clouds. In order to describe the dynamics of the new observations, we then present a transmittance-based model dedicated to layers.

1) Approximation of Sparse-Transmittance Images of Layers: Since there is a loss of information induced by projection in an image plane, several hypotheses are necessary to tackle the reconstruction problem. The troposphere is the lower part of the atmosphere ranging from the sea level up to a bound called tropopause. The layering of atmospheric flow in the troposphere is valid in the limit of horizontal scales much greater than the vertical scale height, thus roughly for horizontal scales greater than $100 \mathrm{~km}$. It is, thus, impossible to guarantee to truly characterize a layered atmosphere with a local analysis performed in the vicinity of a pixel characterizing a kilometer-order scale. Nevertheless, one can still decompose the 3-D space into elements of variable thickness, where only sufficiently thin regions of such elements may really correspond to common layers. Analysis based on such a decomposition presents the main advantage of operating at different atmospheric-pressure ranges and avoids the mix of heterogeneous observations. The analysis of such elements will either be significant of layer dynamics or reveal an average motion significant of thick regions. In this paper, such elements will be defined and called with abusive language "layers."

Let us present the 3-D space decomposition that we chose for the definition of the layers. The $k$ th layer corresponds to the volume lying in between an upper surface $z_{t}^{k}$ and a lower surface $z_{b}^{k}$. In areas where clouds have their top belonging to a given pressure interval, the upper surface corresponds to the height of top of clouds. In other areas, the upper surface is undefined. Note that such a surface is not characterized by a uniform pressure value. In areas with top of clouds belonging to the given pressure interval, the lower surface corresponds to the height of the basis of clouds. In other areas, this surface is undefined.

Now, let us assume that atmosphere is in hydrostatic equilibrium. This assumption provides an excellent approximation for the vertical dependence of the pressure field in the troposphere. Only for small-scale phenomena such as squall lines and tornadoes that it is necessary to consider departures from the hydrostatic balance [17]. We also make the common assumption that integrals of cloud-water density are comparable to integrals of dry-air density. Let us denote pressure and gravity, respectively, by $p$ and $g$. By the vertical integration of the hydrostatic equation

$$
-\rho g=\frac{d p}{d z}
$$

density integrals can be linked to atmospheric-pressure differences

$$
g \int_{z_{b}^{k}}^{z_{t}^{k}} \rho d z=p\left(z_{b}^{k}\right)-p\left(z_{t}^{k}\right)
$$

where pressure may be observed via cloud tracers.

Indeed, top-of-cloud pressure images are routinely provided by the EUMETSAT consortium, the European agency which manages the METEOSAT satellite data. They are derived from a radiative-transfer model using ancillary data, namely, temperature and humidity profiles obtained by analyses or shortterm forecasts. This model simulates the radiation by the top 
of an opaque cloud at different vertical levels, which might be observed by a satellite. The pressure level where the simulated radiation fits best with the observed radiation determines the pressure of the cloud top for the corresponding pixel [23]. Multichannel techniques (using a thermal IR with a water vapor or $\mathrm{CO}_{2}$ absorption channels) enable the determination of the temperature of the top of semitransparent clouds [28], [34] and, thus, their equivalent pressure level (with the help of analyzed or forecast data) independently of foreground or background effects.

The problem now consists in deriving pressure-difference maps characterizing our previously defined layered atmosphere from top-of-cloud pressure observed in the image plane. The membership of top-of-clouds to pressure intervals (consequently to the different layers) is determined by cloudclassification maps. Such classifications, which are based on thresholds of top-of-cloud pressure, are routinely provided by the EUMETSAT consortium. Both, top-of-cloud pressure and classification, constitute intermediate products used to generate the operational segment-based EUMETSAT classification products [23]. Obviously, the discrimination of layers according to classifications based on top-of-cloud potential temperature rather than top-of-cloud pressure would have been better suited to mesoscale. However, such classifications are not currently available. We denote by $C^{k}$ a class corresponding to the $k$ th layer in the altimetric interval $\left[z_{b}^{k}, z_{t}^{k}\right]$. Note that the top-ofcloud pressure image denoted by $p_{\cup}$ is composed of segments of top-of-cloud pressure functions $p\left(z_{t}^{k}\right)$ related to the different layers. That is to say

$$
p_{\cup}=\bigcup_{k} p\left(z_{t}^{k}, \mathbf{s}\right), \quad \mathbf{s} \in C^{k} .
$$

Thus, pressure images of top of clouds are used to constitute sparse-pressure maps of the layer upper boundaries. As in satellite images, clouds lower boundaries are always occluded; we coarsely approximate the missing pressure observations $p\left(z_{b}^{k}\right)$ by an average pressure value observed on top of the layer underneath. In addition, finally, for the $k$ th layer, we define transmittance observations $h^{k}$ as pressure differences

$$
p\left(z_{b}^{k}\right)-p_{\cup}=h^{k} \begin{cases}=g \int_{z_{b}^{k}}^{z_{t}^{k}} \rho d z, & \text { if } \mathbf{s} \in C^{k} \\ \neq g \int_{z_{b}^{k}}^{z_{t}^{k}} \rho d z, & \text { if } \mathbf{s} \in \bar{C}^{k}\end{cases}
$$

which are equal to density integrals on cloudy regions corresponding to the $k$ th layer. Fig. 2 illustrates the different stages of processing necessary to estimate sparse-layer-related transmittance images using pressure images of the top of clouds and cloud-classification maps.

2) Transmittance-Based Model for Atmospheric Layers: Referring to the previous section, layer-transmittance observations are approximated by pressure differences. Layers were defined here as the decomposition of the 3-D space based on top-of-cloud-pressure-classification maps. Moreover, we previously noted that the ICE model of (5) holds for negligible mass exchanges through the planar-integration boundaries related to

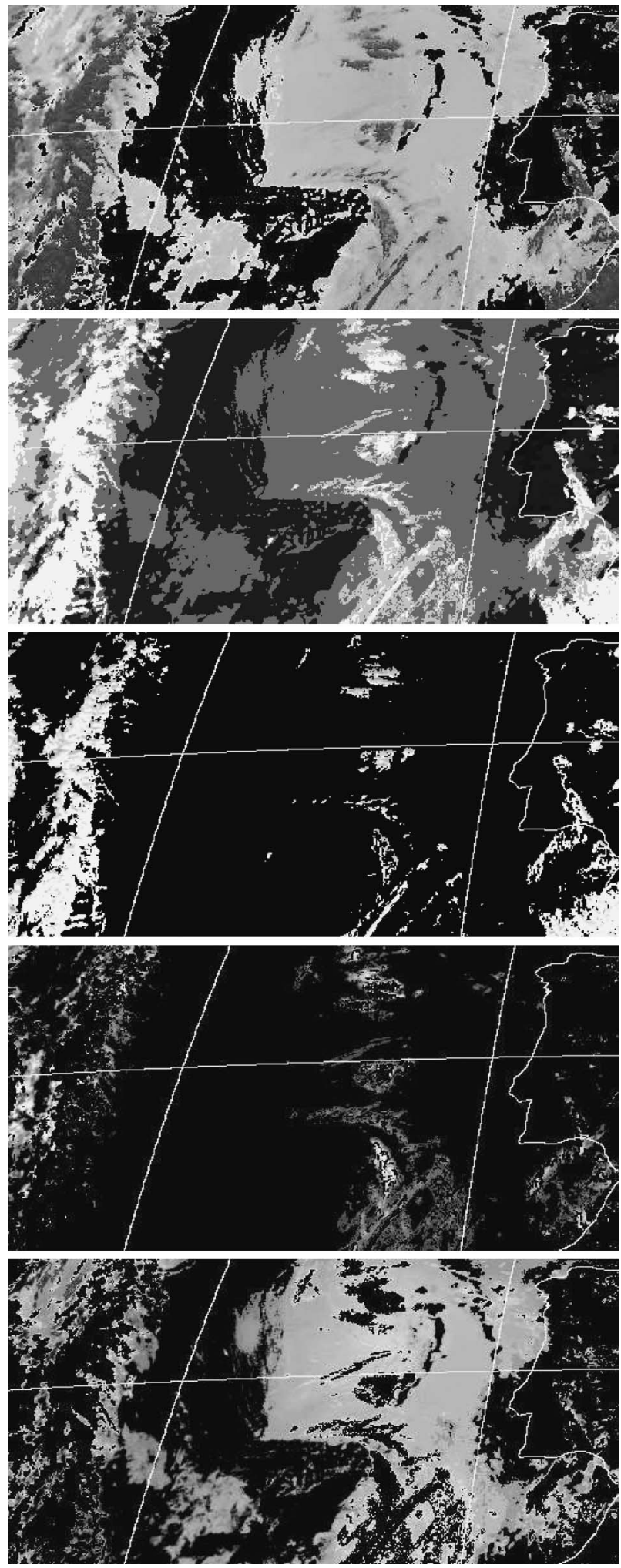

Fig. 2. Recovery of layer transmittances from cloud-top pressure off the Iberian Peninsula. (From top to bottom) Cloud-top-pressure image; classification into low (in dark gray), medium (in light gray), and high (in white) clouds; transmittance of the higher layer; transmittance of the intermediate layer; transmittance of the lower layer. Black regions correspond to missing observations and white lines represent coastal contours, meridians, and parallels (every $10^{\circ}$ ).

transmittance observations. Thus, neglecting mass exchanges via vertical wind on layer boundaries $z_{b}^{k}$ and $z_{t}^{k}$, which are assumed to be locally planar surfaces, the ICE model constitutes 
a physically sound approximation for the layer-transmittance image $h_{\rho}^{k}$ for all $k \in[1, K]$

$$
\frac{\partial h_{\rho}^{k}}{\partial t}+\mathbf{v}^{k} \cdot \nabla h_{\rho}^{k}+h_{\rho}^{k} \operatorname{div}\left(\mathbf{v}^{k}\right)=0
$$

where $K$ is the highest layer index, and $\mathbf{v}^{k}$ corresponds to the density-weighted average horizontal wind related to the $k$ th layer. Note that, as mass exchanges on the layer boundaries has been neglected, this model assumes independent layer motion. As this model relies on the hydrostatic assumption, it is relevant when the aspect ratio of the flow is small, thus, when the horizontal scale of the flow is much greater than the layer depth. Thus, the smaller the layer thickness is, the more accurate the ICE model. Indeed, due to the hydrostatic relation, $h_{\rho}^{k}$ may be viewed as an atmospheric-layer thickness if we neglect density variations. The ICE model then corresponds exactly to shallowwater mass-conservation model [9]

$$
\frac{\partial h^{k}}{\partial t}+\mathbf{v}^{k} \cdot \nabla h^{k}+h^{k} \operatorname{div}\left(\mathbf{v}^{k}\right)=0
$$

where $h^{k}$ is the thickness function of the $k$ th layer. This correspondence will be useful to derive in Section IV-C the complete shallow-water equation system.

Relatively to the different layers, transmittances are sparsely observed only in the presence of clouds. Thus, to tackle the problem of missing observations in regions belonging to $\bar{C}^{k}$ through smoothing div-curl functions and, in the same time, removing data belonging to other levels, we make use of robust estimators. This is presented in the next section.

\section{B. Robust Estimator for Sparse Observations}

A dense estimator dedicated to layer motion should consider simultaneously all cloudy regions belonging to a given layer while discarding the influence of other clouds. For the $k$ th layer, we previously remarked that outside the class " $C^{k}$," the so-defined transmittances $h_{\rho}^{k}$ is not significant at the $k$ th-layer transmittance. Thus, we propose to introduce in (16) a masking operator on unreliable observations. We denote by $\mathbb{I}_{C^{k}}$ the operator which is identity if a pixel belongs to the class, and which returns, otherwise, a fixed value out of the range taken by $h_{\rho}^{k}$. Thus, applying this new masking operator in (16), we obtain the robust data term

$$
\begin{array}{r}
J_{d}\left(\mathbf{v}, h_{\rho}^{k}\right)=\int_{\Omega} \phi_{d}\left\{\operatorname { e x p } \operatorname { d i v } ( \tilde { \mathbf { v } } ^ { k } ( \mathbf { s } ) ) \left(\left[\tilde{h}_{\rho}^{k}(\mathbf{s}) \nabla \operatorname{div}\left(\tilde{\mathbf{v}}^{k}(\mathbf{s})\right)\right.\right.\right. \\
\left.\left.\left.+\nabla \tilde{h}_{\rho}^{k}(\mathbf{s})\right]^{\top} \mathbf{v}^{\prime k}(\mathbf{s})+\tilde{h}_{\rho}^{k}(\mathbf{s})\right)-\mathbb{I}_{C^{k}}\left(h_{\rho}^{k}(\mathbf{s})\right)\right\} d \mathbf{s}
\end{array}
$$

where $\mathbf{v}^{k}=\tilde{\mathbf{v}}^{k}+\mathbf{v}^{\prime k}$ corresponds to the density-weighted average horizontal wind related to the $k$ th layer. The robust div-curl smoothing term (17) is conserved. The masking procedure, together with the use of robust penalty function on the data term, allows one to discard implicitly the erroneous observation from the estimation process. The robust penality function related to the smoother handles discontinuous-motion fields induced, for example, by the possible spatial heterogeneity of layers. It is important to outline that, for the $k$ th layer, the method always provides a motion vector on all points $s$ of the image domain. Areas outside the cloud class " $C^{k}$ " correspond to an interpolated wind field.

The dedicated method, which has been presented, proposes to solve the 3-D motion-estimation problem by the inference of dense density-weighted average horizontal wind fields related to different atmospheric strata. The motion extraction is done by fitting an image-adapted mass-conservation model to pressure observations. However, due to the complexity of atmospheric motion observed at mesoscale and because of noise and sparse observations, motion estimation may lack in accuracy. Thus, the aim of the following section is to devise a method allowing us to overcome such a limitation.

\section{Two-Stage Mesoscale Motion Estimator}

In order to enhance the estimation accuracy, we propose an original two-stage estimation scheme for mesoscale motion characterization. In a first stage, acting at the upper range of mesoscale dynamics, by the introduction of correlation-based constraints and the use of a priori information on atmosphere dynamics, the proposed approach guides the variational estimation process toward a large displacement solution. In a second step, acting at the lower range of mesoscale dynamics, the solution is refined by an incremental process.

The proposed large-displacement estimation step is an alternative approach to common multiresolution used in the context of differential-motion estimator. Unlike a multiresolution scheme, our approach relies on a unique representation of the full-resolution image. Thus, the proposed method tackles the nonlinear estimation problem with neither making successive approximations in the calculation of interpolated images nor restricting itself to the characterization of large displacements of sufficiently large structures. The proposed method takes advantage of a matching formulation of the motion-estimation problem within a differential framework appropriate for global smoothing. More explicitly, superficial large displacement of both fine and large clouds can be extracted with correlationbased methods for sufficiently contrasted regions which possess a low spatial variability. Even in the difficult case where the layer underneath moves in an opposite direction, large displacements of fine and very sparse structures can be recovered. The idea is thus to avoid the multiresolution coarse-estimation levels by introducing a collection of correlation-based vectors in a differential scheme for the estimation of a large-scale densemotion field.

Furthermore, we propose that the estimation scheme incorporates a priori physical knowledge on fluid dynamical evolution. Indeed, in order to preserve spatio-temporal consistency of displacement estimates at large scales, a simplified Navier-Stokes dynamical model is adapted to images depicting atmospheric layers. A dense displacement field is predicted by time integration of a physical dynamical model. The propagated field is then introduced in the estimation process as a spatio-temporal smoother applying at large scales. A priori information introduced with such a dynamic model may enhance significantly 
the quality of estimates particularly in the case of noisy and incomplete observations.

Let us remark that correlation-based displacement vectors are significant of motion on the upper boundary of layers. Thus, these measurements slightly differ from density-weighted average winds. Thus, we choose to rely on correlation-based measurements only at large scales and to rely on the ICE data model (23) at finer scales. In an analogous way, since atmosphere dynamics appear to be more complex at finer scales, we choose to perform spatio-temporal smoothing only at large scales, employing a dynamical model describing motion evolution on the layer upper boundary. Spatio-temporal smoothing is therefore acting on the same resolution level as the correlation-based constraints. This solution is then refined in a second stage toward the estimation of density-weighted average winds based exclusively on the ICE data model (23) and on spatial smoothing.

\section{A. Two-Stage Estimation Scheme}

The displacement field $\mathbf{v}^{k}$ is decomposed into a displacement field characterizing large scales $\overline{\mathbf{v}}^{k}$ and an additive displacement field $\breve{\mathbf{v}}^{k}$ related to finer scales

$$
\mathbf{v}^{k}=\overline{\mathbf{v}}^{k}+\breve{\mathbf{v}}^{k}
$$

Keeping notations of Section II-C, we define for the estimation of variable $\mathbf{v}$ the new functional

$$
\begin{aligned}
J\left(\overline{\mathbf{v}}^{k}, \breve{\mathbf{v}}^{k}\right)=J_{d}\left(\overline{\mathbf{v}}^{k}+\breve{\mathbf{v}}^{k}, h_{\rho}^{k}\right)+\alpha J_{r}\left(\overline{\mathbf{v}}^{k}+\breve{\mathbf{v}}^{k}\right) \\
+\beta J_{p}\left(\overline{\mathbf{v}}^{k}, \overline{\mathbf{v}}_{p}^{k}\right)+\gamma J_{c}\left(\overline{\mathbf{v}}^{k}, \overline{\mathbf{v}}_{c}\right)
\end{aligned}
$$

where $J_{c}($.$) and J_{p}($.$) are energy functions constraining large-$ scale displacements $\overline{\mathbf{v}}^{k}$ to be close to a sparse correlationbased vector field $\overline{\mathbf{v}}_{c}$ and to be consistent with a physically sound prediction $\overline{\mathbf{v}}_{p}^{k}$ relying on Navier-Stokes equations. In the previous expression, $\gamma$ and $\beta$ denote weighting factors. Functionals $J_{c}($.$) and J_{p}($.$) will be further detailed in the$ following sections.

The optimization problem is conducted sequentially. In a first step, large displacements $\overline{\mathbf{v}}^{k}$ are estimated while the variable $\breve{\mathbf{v}}^{k}$ is fixed to zero in (26). Here, an analogous version of the alternate multigrid minimization scheme that is proposed in [7] and [27] has been implemented, the only difference being in the internal Gauss-Seidel solver for variable $\overline{\mathbf{v}}^{k}$ estimation. Once the minimum has been reached, a second refinement step is launched: The small-scale displacement increments $\breve{\mathbf{v}}^{k}$ are estimated while the large-displacement variable $\overline{\mathbf{v}}^{k}$ is frozen in (26). More explicitly, $\overline{\mathbf{v}}^{k}$ is used to derive a motioncompensated expression of the data model (24) and to express the div-curl in terms of the displacement increment $\breve{\mathbf{v}}^{k}=\mathbf{v}^{k}-$ $\overline{\mathbf{v}}^{k}$ (17). This incremental motion estimate relies neither on correlation-based vectors nor on spatio-temporal constraints, which only apply at large scales. The displacement-increment estimate is again obtained by alternate multigrid minimization.

Note that, in the case $\alpha, \beta, \gamma \gg 1$, the energy minimization leads to a large displacement field, which can be seen as a phys- ically sound spatio-temporal interpolation of the correlationbased vectors.

Sparse-correlation measurements need to be introduced as a constraint in the variational scheme employed for motion estimation at large scales. We define the function implementing this constraint in the following section. We will then derive from filtered Navier-Stokes equations a simplified dynamical model for spatio-temporal smoothing at large scales.

\section{B. Correlation Within a Variational Estimation Scheme}

In order to obtain a dense estimation of large-scale displacements fitting a sparse-correlation-based displacement field (9), we define a functional where the $i$ th correlation-based vector $\overline{\mathbf{v}}_{c}^{i}=\left(\bar{u}^{i}, \bar{v}^{i}\right)$ located at the point $\mathbf{s}^{i}=\left(x^{i}, y^{i}\right)$ influences its neighborhood according to a shifted 2-D Gaussian $\mathcal{N}^{i}\left(\mathbf{s}^{i}-\mathbf{s}\right)$ of variance $\sigma$ related to the correlation window size

$$
J_{c}\left(\overline{\mathbf{v}}^{k}, \overline{\mathbf{v}}_{c}\right)=\int_{\Omega} \sum_{i=1}^{L} \mathbb{I}_{C^{k}}\left(g^{i} \mathcal{N}^{i}\left(\mathbf{s}^{i}-\mathbf{s}\right)\left|\overline{\mathbf{v}}_{c}^{i}-\overline{\mathbf{v}}^{k}(\mathbf{s})\right|^{2}\right) d \mathbf{s}
$$

where $L$ and $g^{i}$ denote, respectively, the number of correlation vectors and confidence factors which were fixed to the values taken by the dissimilarity function. In the previous expression, the masking operator $\mathbb{I}_{C^{k}}()$ was introduced, since the joint use of correlation and optical-flow is not possible in regions with no image observations.

For sake of completeness, in Appendix I, we briefly detail the modifications induced by the introduction of $J_{c}($.$) in$ the multigrid Gauss-Seidel solver used for the minimization of (26).

\section{Physical A Priori for Spatio-Temporal Smoothing}

1) Spatio-Temporal Consistency: The functional $J_{p}($.$) aims$ at constraining motion field at large scales to be consistent with a physically predicted wind field. We simply define this function as a quadratic distance between the estimated field $\overline{\mathbf{v}}^{k}$ and the dense propagated field $\overline{\mathbf{v}}_{p}^{k}=\left(\bar{u}_{p}, \bar{v}_{p}\right)$

$$
J_{p}\left(\overline{\mathbf{v}}^{k}, \overline{\mathbf{v}}_{p}^{k}\right)=\int_{\Omega}\left\|\overline{\mathbf{v}}_{p}^{k}(\mathbf{s})-\overline{\mathbf{v}}^{k}(\mathbf{s})\right\|^{2} d \mathbf{s} .
$$

This approach constitutes an alternative to the spatiotemporal smoother defined in [40] and is, to some extent, similar to the temporal constraint introduced in [32]. In [40], the temporal derivative of the velocity vectors is constrained to be weak, which is not consistent with fluid-flow dynamics. The smoothing proposed in [32] constrains the estimated vorticity to be close to a prediction. Our propagation model includes a 2-D divergence component, which is equal to zero only for incompressible 2-D flows. As it is detailed as follows, our approach extends this scheme to the spatio-temporal smoothing of the full-velocity field in the case of 3-D geophysical flows driven by a shallow-water evolution law. The modifications induced by the introduction of $J_{p}($.$) in the multigrid Gauss-Seidel solver$ are detailed in Appendix I. 
2) Large Eddy Shallow-Water Prediction Model: Dynamical models describing wind-field evolution are needed here for the prediction at time $t+1$ of a large-scale motion field $\overline{\mathbf{v}}_{p}^{k}$ using the previous estimation performed for the $k$ th layer between time $t-1$ and $t$. It is important to distinguish this dynamical model from the layer-transmittance-based model proposed in Section III-A. Indeed, the latter is analogous to a likelihood term acting at large and fine scales while the former only provides an a priori knowledge, conditioning the temporal consistency of the estimates at large scales.

As atmosphere dynamics is governed by fluid-flow laws, we may rely on Navier-Stokes equations in order to derive simplified dynamical models for short-time propagation of layer mesoscale motion. However, predicting atmospheric dynamics at the pixel scale on the order of a few kilometers requires the accurate knowledge of dense 3-D maps characterizing atmospheric-state variables. As our aim is restricted to spatiotemporal smoothing at large scales, we rely instead on the filtered Navier-Stokes equations predicting wind fields at scales on the order of $100 \mathrm{~km}$, that is to say, where rotational motion dominates divergent motion.

For characteristic scales on the order of $100 \mathrm{~km}$, the layering of the atmosphere constitutes a good approximation. We assume incompressibility within the layers. That is to say, we consider a constant density $\rho_{0}$, which implies under hydrostatic balance that horizontal divergence is weak. Let us remark that this incompressibility simplification, which underlies a shallow-water system, is reasonable, while it may be erroneous for finer scales. Denoting, respectively, by $\mathcal{F}, \mathcal{T}=\left(\mathcal{T}_{\bar{u}}, \mathcal{T}_{\bar{v}}\right)^{\top}$, $\phi, \Upsilon$, and $a$ the viscous forces, turbulent viscosity dissipation at subgrid scales [12], latitude, Earth angular velocity, and radius, the horizontal momentum equations read thus [17]

$$
\left\{\begin{array}{l}
\frac{d \bar{u}}{d t}-\frac{\bar{u} \bar{v} \tan \phi}{a}+\frac{\bar{p}_{x}}{\rho_{0}}=+2 \Upsilon \bar{v} \sin \phi+\mathcal{F}+\mathcal{T}_{\bar{u}} \\
\frac{d \bar{v}}{d t}+\frac{\bar{u}^{2} \tan \phi}{a}+\frac{\bar{p}_{y}}{\rho_{0}}=-2 \Upsilon \bar{u} \sin \phi+\mathcal{F}+\mathcal{T}_{\bar{v}}
\end{array}\right.
$$

where the unknown quantities are the pressure function $\bar{p}$ and the horizontal wind fields $(\bar{u}, \bar{v})$ filtered with a Gaussian kernel function of standard deviation equal to $\Delta_{x}=100 \delta_{p}^{-1}$, where $\delta_{p}$ denotes the image-pixel resolution in kilometers. Adaptation of filtered Navier-Stokes equations to mesoscale atmospheric short-time evolution relies on the scale analysis presented in Appendix II. It enables us to neglect the curvature terms and viscous effects. Note that the wind field $(\bar{u}, \bar{v})$ defined in (29) is different from the wind field $\overline{\mathbf{v}}^{k}$. The former quantity is related to 2-D winds at a physical point of the 3-D space while the latter corresponds to a spatially filtered density average horizontal wind $\mathbf{v}^{k}$ related to the $k$ th layer. To recover the latter quantity, imposing incompressibility in the hydrostatic relation (18), an integrated form of the momentum equations is produced by vertical integration in-between the $k$ th-layer boundaries. It yields to the shallow-water momentum equations [9]. However, a simplified model of these equations can be obtained when relying on the assumption that, at large scales, horizontal motion on the upper boundary of layers is similar to the density-weighted average horizontal wind [19]. In other words, the simplification assumes that filtered horizontal winds are homogenous within the layer and thus neglect their vertical derivatives. Moreover, note that so-defined winds related to layer boundaries are consistent with the previously introduced correlation-based displacement vectors. Thus, by adding the filtered mass-conservation model obtained by filtering spatially (23), we form a simplified shallow-water system supplemented by Coriolis effects, which reads

$$
\left\{\begin{array}{l}
\overline{\mathbf{v}}_{t}^{k}+\nabla\left(\overline{\mathbf{v}}^{k}\right) \overline{\mathbf{v}}^{k}-g \boldsymbol{\nabla} \bar{h}^{k}+\left[\begin{array}{cc}
0 & -1 \\
1 & 0
\end{array}\right] f^{\phi} \overline{\mathbf{v}}^{k}=\mathcal{T} \\
\bar{h}_{t}^{k}+\overline{\mathbf{v}}^{k} \cdot \nabla \bar{h}^{k}+\bar{h}^{k} \bar{D}^{k}=0
\end{array}\right.
$$

where $\bar{h}^{k}$ and $f^{\phi}=2 \Upsilon \sin \phi$ denote, respectively, the filtered height functions and the Coriolis coefficient. The induced turbulent dissipation can be approached by subgrid models proposed in large-eddy simulation literature. The simplest one is the well-known Smagorinsky subgrid model. Assuming isotropic turbulence in agreement with Kolmogorov "K41" theory [12], the subgrid model proposes a turbulent viscosity dissipation implemented with a weighted Laplacian operator [35]. For a vorticity-based LES formulation, we may rely on similar enstrophy-based subgrid models relying on Taylors vorticity transfer and dissipation by small-scales theory [38]. Consequently, denoting by $\nu_{s}$, a subgrid scale viscosity coefficient $\mathcal{T}$ is replaced by a diffusion term in the above momentum equations. Let us denote the vorticity by $\bar{\zeta}^{k}=\operatorname{curl}\left(\overline{\mathbf{v}}^{k}\right)$ and the divergence by $\bar{D}^{k}=\operatorname{div}\left(\overline{\mathbf{v}}^{k}\right)$. The previous system may be expressed in its vorticity-divergence form

$$
\left\{\begin{array}{l}
\bar{\zeta}_{t}^{k}+\overline{\mathbf{v}}^{k} \cdot \boldsymbol{\nabla} \bar{\zeta}^{k}+\left(\bar{\zeta}^{k}+f^{\phi}\right) \bar{D}^{k}=\nu_{s} \Delta \bar{\zeta}^{k} \\
\bar{D}_{t}^{k}+\overline{\mathbf{v}}^{k} \cdot \nabla \bar{D}^{k}+\left(\bar{D}^{k}\right)^{2}-2|J|-g \Delta \bar{h}^{k}+f \bar{\zeta}^{k}=\nu_{s} \Delta \bar{D}^{k} \\
\bar{h}_{t}^{k}+\overline{\mathbf{v}}^{k} \cdot \nabla \bar{h}^{k}+\bar{h}^{k} \bar{D}^{k}=0
\end{array}\right.
$$

where $|J|$ is the determinant of the Jacobian matrix of variables $\left(\bar{u}^{k}, \bar{v}^{k}\right)$, and where we have chosen the enstrophy-based subgrid-scale model proposed in [24], [31], and [41]

$$
\nu_{s}=\left(C \Delta_{x}\right)^{2}\left|\bar{\zeta}^{k}\right|
$$

where $C$ has a universal value of 0.17 . In the two previous shallow-water formulations, dynamical models predict the evolution of three variables, which may depend on each other. One of the major difficulties is induced by the fact that variable $h^{k}$ is derived only for cloudy regions corresponding to the $k$ th layer. Therefore, variable $h^{k}$ and, thus, all unknowns can only be propagated on a sparse spatial support. However, in opposition to the classical formulation, the vorticity-divergence equations provide a dynamical model for which the vorticity evolution is independent of variable $h^{k}$ and for which the divergence evolution depends only weakly on variable $h^{k}$. Based on the realistic assumption that, at large scales, divergence is weak almost everywhere and comparable to noise, we propose to simplify the divergence dynamical model in order to make it independent of variable $h^{k}$. Divergence $\bar{D}$ is assumed to be driven by a Gaussian random function of standard deviation equal to $\Delta_{x} t / 2$ with stationary increments (i.e., a standard Brownian motion). As a consequence, neglecting probabilities of increments greater than $\Delta_{x} t / 6$, divergence expectation 
asymptotically obeys to a heat equation of diffusion coefficient equal to $\left(\Delta_{x} / 6 \sqrt{2}\right)^{2}$ [6]. The simplified vorticity-divergence model reads

$$
\left\{\begin{array}{l}
\bar{\zeta}_{t}^{k}+\overline{\mathbf{v}}^{k} \cdot \nabla \bar{\zeta}^{k}+\left(\bar{\zeta}^{k}+f^{\phi}\right) \bar{D}^{k}=\left(C \Delta_{x}\right)^{2}\left|\bar{\zeta}^{k}\right| \Delta \bar{\zeta}^{k} \\
\bar{D}_{t}^{k}=\left(\frac{\Delta_{x}}{6 \sqrt{2}}\right)^{2} \Delta \bar{D}^{k}
\end{array}\right.
$$

The curl and divergence completely determine the underlying 2-D velocity field, and the current velocity estimate can be recovered from these quantities up to a laminar flow. Indeed, denoting the orthogonal gradient by $\nabla^{\perp}=(-\partial / \partial y, \partial / \partial x)^{\top}$, the Helmholtz decomposition of the field into a sum of gradients of two potential functions is expressed as

$$
\overline{\mathbf{v}}^{k}=\nabla^{\perp} \Psi+\nabla \Phi+\overline{\mathbf{v}}_{\text {har }}^{k}
$$

where $\overline{\mathbf{v}}_{\text {har }}^{k}$ is a harmonic transportation part $\left(\operatorname{div} \overline{\mathbf{v}}_{\text {har }}^{k}=\right.$ $\operatorname{curl} \overline{\mathbf{v}}_{\text {har }}^{k}=0$ ) of the field $\overline{\mathbf{v}}^{k}$, and where the stream function $\Psi$ and the velocity potential $\Phi$ correspond to the solenoidal and the irrotational part of the field. The latter terms are linked to divergence and vorticity through two Poisson equations. Expressing the solution of both equations as a convolution product with the 2-D Green kernel $G$ associated with the Laplacian operator: $\Psi=G * \zeta, \quad \Phi=G * D$, the whole velocity field can be recovered with the equation

$$
\overline{\mathbf{v}}^{k}=\nabla^{\perp}\left(G * \bar{\zeta}^{k}\right)+\nabla\left(G * \bar{D}^{k}\right)+\overline{\mathbf{v}}_{\mathrm{har}}^{k}
$$

which can be efficiently solved in the Fourier domain. The harmonic transportation component $\overline{\mathbf{v}}_{\text {har }}^{k}$ is recovered by subtracting to the field $\overline{\mathbf{v}}^{k}$ its solenoidal and irrotational parts.

Let us sum up this prediction process. The vorticity and the divergence fields are developed in time in between consecutive image frame using a discretized form of (33) and time increments $\delta t$. After each time increment, assuming $\overline{\mathbf{v}}_{\text {har }}^{k}$ constant within each frame interval, (35) is used to update the velocity $\overline{\mathbf{v}}^{k}$ needed by (33) with the current vorticity and divergence estimates.

To avoid instability, a semiimplicit time-discretization scheme is used to integrate forward (33). Classical centered finite-difference schemes are used for the curl and divergence discretization. Geometrical deformations due to slant view effects are neglected for geostationary satellite observations at tropical and temperate latitudes in order to perform spatial discretization using directly the pixel grid. Let us note that this equation system describes the dynamics of physical quantities expressed in standard units. Thus, a dimension factor appears in front of the Coriolis factor when this equation system is discretized on a pixel grid with velocity expressed in pixel per frame.

To solve the linear system associated with the semiimplicit discretization scheme, the matrix has been constrained to be diagonally dominant, which is a sufficient condition for a wellconditioned inversion problem. This condition reads $1 / \delta t \geq$ $\max _{s}\left(\left|\bar{u}^{k}\right|+\left|\bar{v}^{k}\right|-\left|\bar{D}^{k}\right|\right)$.

Finally, the dynamical model time integration is done independently for each layer. This procedure results in a predicted average horizontal wind field $\overline{\mathbf{v}}_{p}^{k}$ related to each layer.

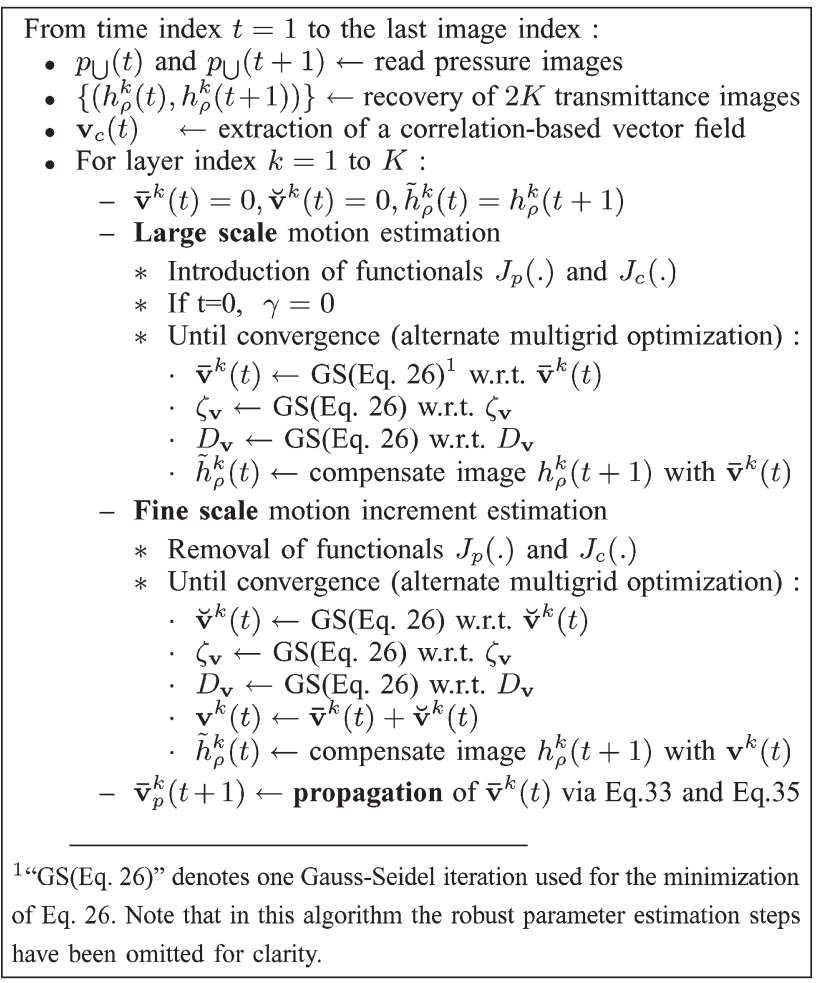

Fig. 3. Global flowchart for atmospheric-motion estimation.

The complete algorithm for atmospheric motion estimation is summarized in Fig. 3.

\section{EXPERIMENTAL EVALUATION}

In this section, we assess the respective merits of the multilayer model used for motion estimation, which has been presented in Section III-B, of the collaborative framework introduced in Section IV-B and of the spatio-temporal smoother presented in Section IV-C.

Because of the extent and the variety of scales of geophysical flows, ground-truth data cannot be collected at mesoscale in a 3-D space. An alternative recourse is to make a comparison with so-called "analyzed winds" produced by data assimilation. However, they are model-dependent and cannot be considered as proper ground truth. Moreover, analyzed winds are calculated at coarse resolution for the given pressure levels. Because of turbulence properties, motion might be slightly different when observed at finer spatial and temporal resolution.

As a consequence, the assessment of the proposed transmittance-based model for atmospheric layers is mainly conceptual and relies on physical justifications. Nevertheless, as we shall see in Section V-B, qualitative comparisons performed on a real METEOSAT image sequence demonstrate the enhancements brought by the transmittance-based model and the potential of the entire method.

However, for an exhaustive evaluation of the spatio-temporal smoother and the introduction of correlation-based matchings in a variational scheme, we propose instead to rely on a simulated flow. A direct numerical simulation (DNS) of a 2-D, incompressible, and highly turbulent flow has been used 


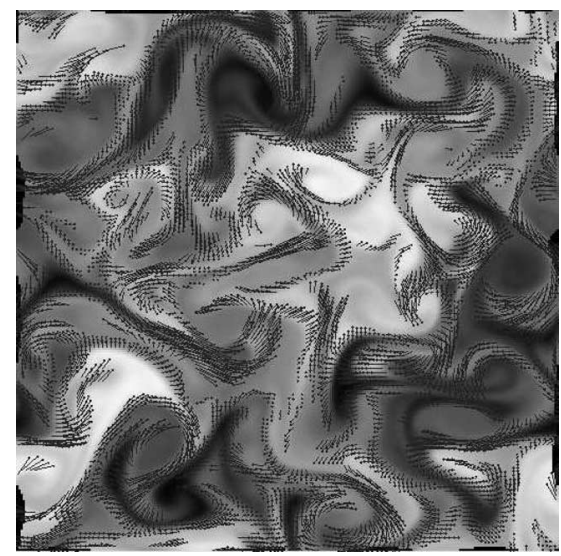

Fig. 4. Velocity constraints and fluid imagery for a 2-D flow. Velocity vectors provided by the DNS, which have been selected as constraints, are superimposed on the image.

to generated an image sequence depicting the motion of a continuous scalar field. The main interest of such a synthetic image sequence is the knowledge of the exact solution along time and for a wide range of spatial scales including pixel resolution.

\section{A. Synthetic 2-D Turbulent Flows}

The sequence of scalar images of $256 \times 256$ pixels, together with the true vector fields generated by the DNS of an incompressible 2-D flow possessing a Reynolds number of 30000 , were provided by the Laboratory of Fluid Mechanics of Cemagref (center of Rennes, France). Two dimensionality was chosen to simplify the simulation. The latter assumption, which involves a nondivergent flow, is consistent with the fact that divergence was considered weak and comparable to noise. Thus, the transmittance-conservation model reduces, in this special case, to the classical OFC data model (11). Note that, as divergence vanishes, the spatio-temporal smoothing constrains only vorticity to be coherent in time.

In order to experiment our method with correlation-based vectors with different noise levels, the correlation-based vectors have been substituted by DNS vectors contaminated by additive Gaussian noise. As correlation techniques only operate on contrasted regions, vector constraints were attached to regions with sufficient gradient. To be realistic with correlation measurements, DNS vectors have been subsampled in those regions. Fig. 4 presents the noise-free DNS velocity vectors, which have been selected as correlation measurements. They are superimposed on the scalar image. As for this experimental case, the propagation model and the OFC data model (11) are valid for both large and fine scales; the temporal and correlation-based constraints were maintained during the two estimation stages.

Based on the nonnoisy correlation constraints defined previously, we first compare our two-stage collaborative scheme to the fluid-flow-dedicated multiresolution approach described in [7]. In Fig. 5, it clearly appears that the multiresolution approach hardly estimates fine turbulent structure, while the collaborative method manages to characterize most of the vor-

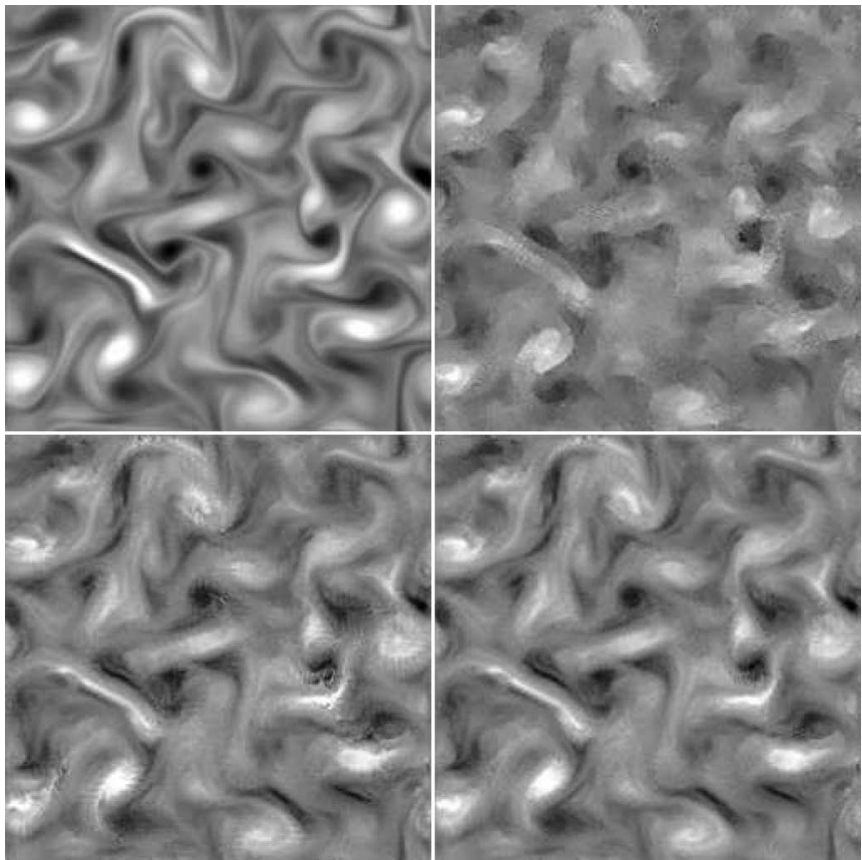

Fig. 5. Comparison in the image domain of multiresolution and collaborative schemes in the case of a 2-D flow. (Top left) Vorticity provided by the DNS. (Top right) Vorticity estimation by the fluid-flow-dedicated multiresolution approach of [7]. (Bottom) Vorticity estimation after (left) the first and (right) the second level of the collaborative scheme.

ticity field structures. Indeed, in scalar imagery, low-contrast regions correspond to high-vorticity areas. Thus, the multiresolution technique suffers from a lack of information in those crucial regions. In addition, incorporating motion constraints in contrast areas around vortices reduces the degree of freedom of the solution and, thus, considerably enhances the estimated motion field. The way the two levels of estimation operate is illustrated in Fig. 5: the global shape of the vorticity field is retrieved in the first level, while in the second level, finer errors are removed and the solution is refined. For a better visual inspection, Fig. 6 (top) reveals the enhancement brought by the collaborative method on a typical horizontal slice of the image. Spectra of the velocity are shown in Fig. 6 (bottom) for the DNS, for the multiresolution, and for the collaborative approach. It can be observed that, for both large and small wavelengths, the collaborative approach exhibits a better representation of the velocity spectrum. This spectral analysis shows that the method improves the main shape of the motion field while performing a better estimation of fine structures.

In order to evaluate the robustness of the collaborative method to inaccurate constraints, Gaussian noise of zero mean and increasing variance has been added to the true velocity vectors provided by the DNS. An example of noisy correlation constraints superimposed on a related image is displayed in Fig. 7. This can be compared to the noise-free vectors presented in Fig. 4. To quantify the estimation accuracy, root-mean-square (rms) errors on vorticity are indicated in the legend of Fig. 7. As the flow is nondivergent, owing to Helmholtz decomposition, the curl of the flow completely determines the underlying 2-D 

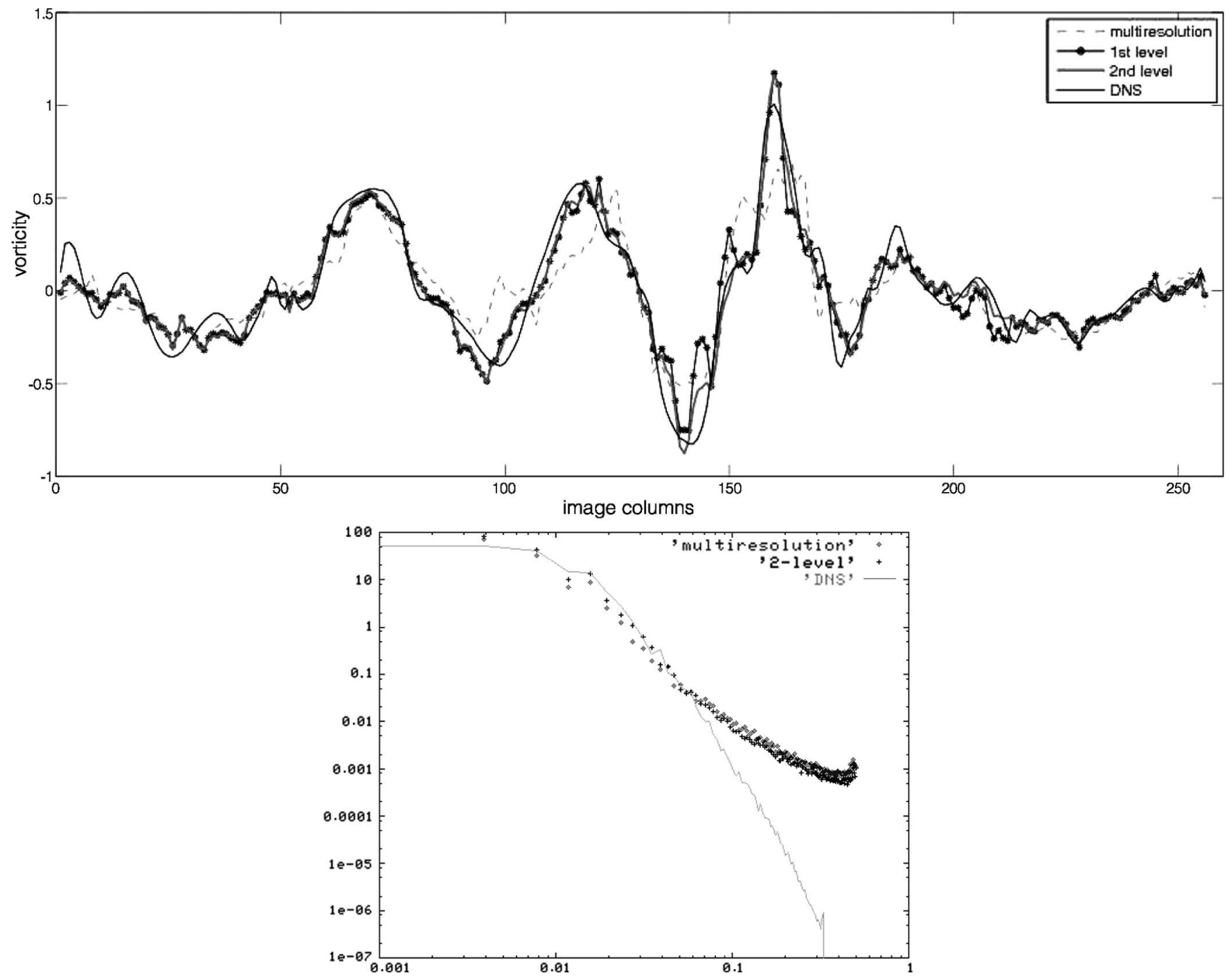

Fig. 6. Comparison in the image and spectral domains of multiresolution and collaborative schemes in the case of a 2-D flow. (Top) On a particular horizontal slice of the image, comparison of vorticity estimated by the multiresolution and by each level of the collaborative scheme with vorticity provided by the DNS. (Bottom) Comparison in "log-log" coordinates of average horizontal velocity spectra estimated by the two different approaches and by the DNS.

flow up to a harmonic transportation component. Therefore, rms errors on vorticity reveal the global accuracy of the estimated field. In Fig. 7, we can visually inspect the influence of noise on the estimated solution for a particular horizontal slice of the image and for the global image domain by referring to rms values. It clearly appears that, even in the presence of noise, motion estimation is better achieved by our collaborative scheme than by a classical multiresolution approach.

Spatio-temporal smoothing benefits are assessed in the image and in the spectral domain. The global enhancement on estimation accuracy brought for both multiresolution and collaborative methods is shown in Fig. 8. There is obviously no improvement for the first pair of images for which only a null initialization is available. It is worth noting that the incorporation of temporal consistency is all the more advantageous for small scales. This can be visualized by comparing the spectra of Fig. 8. Indeed, the temporally regularized spectrum is closer to the true one for high frequencies. Therefore, if large vortices are sufficiently well estimated, then introducing a vorticity temporal consistency can significantly improve the estimation of small vortex structures. In other words, the better large scales are estimated, the better fine scales are inferred by spatio-temporal smoothing.

\section{B. METEOSAT Satellite Image Sequence}

We then turned to qualitative comparisons on a real meteorological image sequence.

The benchmark data was composed by a sequence of 18 METEOSAT second-generation images, showing top-ofcloud pressures with a corresponding cloud-classification sequence. The $512 \times 512$ pixel images cover an area over the North Atlantic Ocean, off the Iberian Peninsula, during part of one day (June 5, 2004) at a rate of one image every $15 \mathrm{~min}$. The spatial resolution is $3 \mathrm{~km}$ at the center of the whole Earth image disk. Clouds from a cloud classification were used to segment images into three broad layers, at low, intermediate, and 

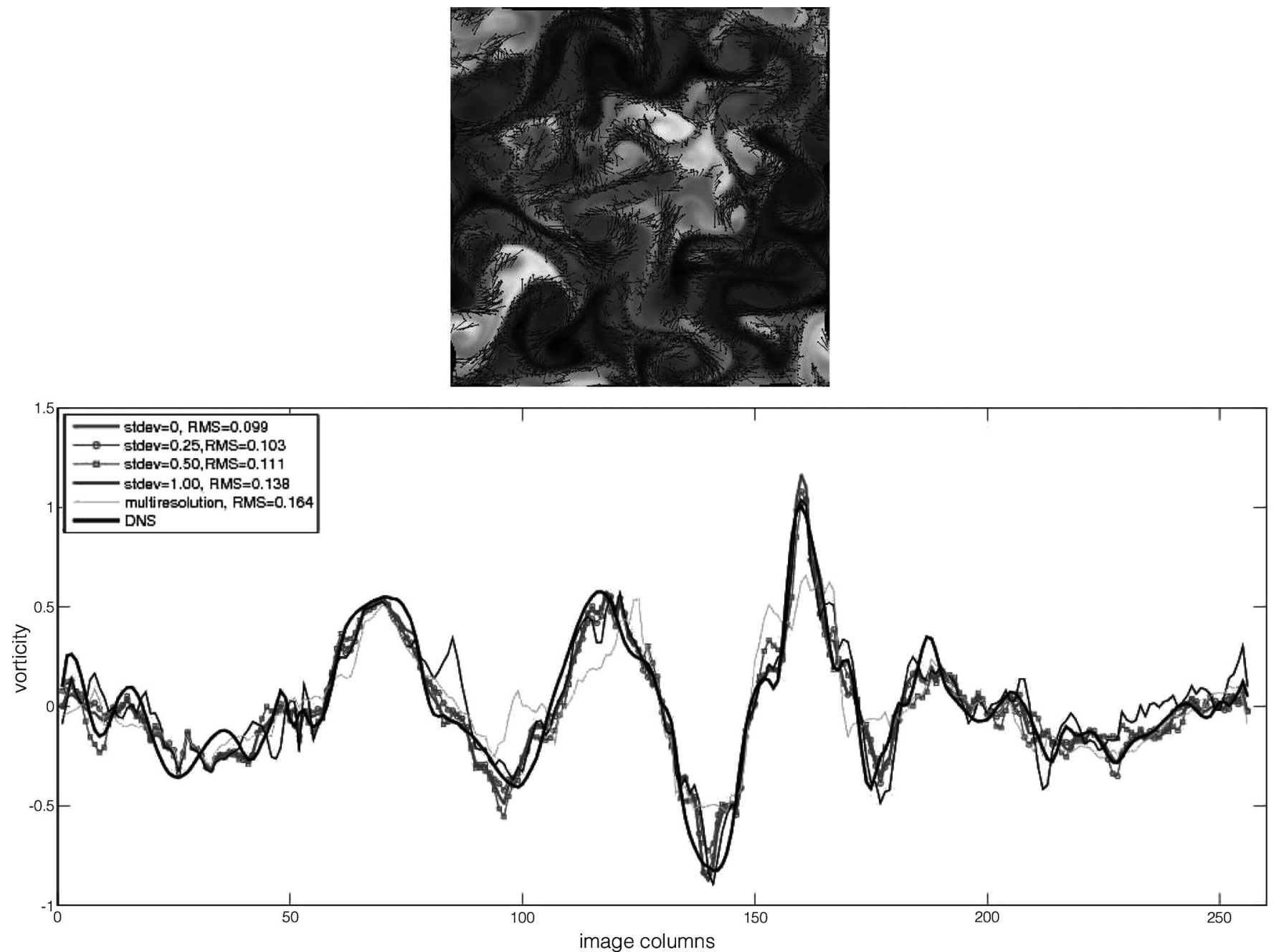

Fig. 7. Influence of noise. (Top) Gaussian noise $\mathcal{N}(0,1)$ has been added to the true velocity vectors provided by the DNS. The subparts, which have been selected as constraints, are superimposed on the image. (Bottom) On a typical horizontal slice of the image, vorticity estimates and rms vorticity error for increasing noise in comparison to the multiresolution approach.
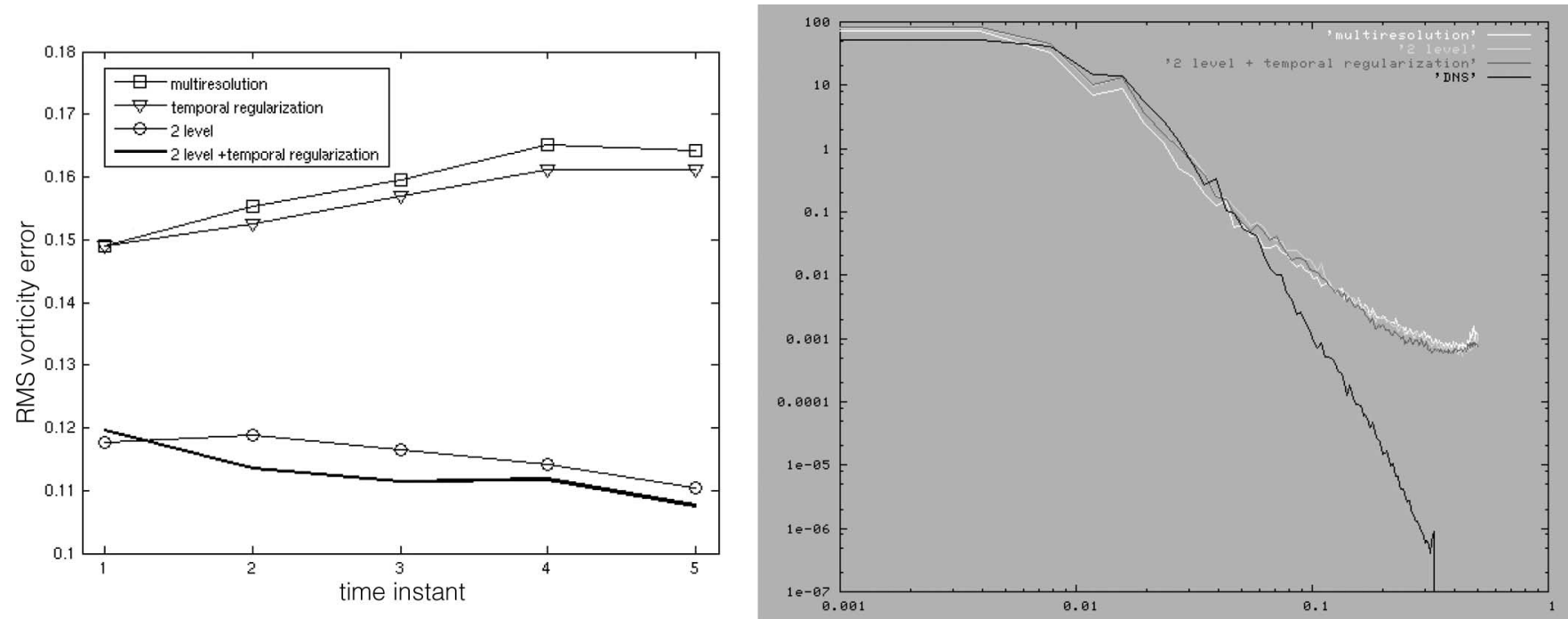

Fig. 8. Evaluation of spatio-temporal smoothing in the case of 2-D flows. (Left) RMS vorticity errors calculated on five consecutive images for the multiresolution approach, the collaborative scheme constrained by vectors contaminated by a Gaussian noise $\mathcal{N}(0,1 / 2)$ combined or not with spatio-temporal smoothing. (Right) Comparison in the spectral domain in "log-log" coordinates of average horizontal velocity estimated by the different methods and provided by the DNS. 

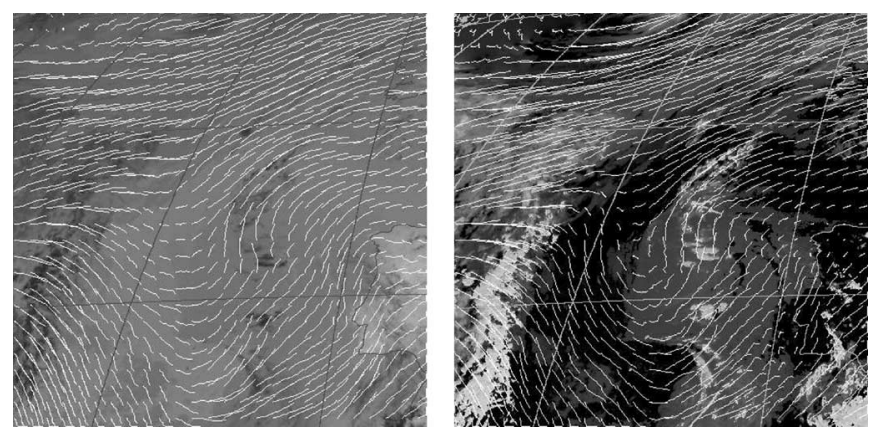

Fig. 9. Trajectories for the multiresolution technique based on (left) the "infrared-based" model [7] and (right) our "transmittance-based" model considering a single layer have been reconstructed based on wind fields estimated from a sequence of 18 METEOSAT images above the North Atlantic Ocean, off the Iberian Peninsula. The trajectories are superimposed on the final infrared or transmittance image of the sequence. Gray lines represent coastal contours, meridians, and parallels (every $10^{\circ}$ ).

high altitudes. ${ }^{1}$ Applying the methodology described in Section III-A, transmittance images were derived from pressure images for these three layers. Trajectories reconstructed from the estimated wind fields provide a practical visualization tool to assess the quality of the estimation in time and space. Therefore, for visualization issues, trajectories have been reconstructed using a fourth-order Runge-Kutta integration method, as suggested in [7].

Results obtained without discarding clear-sky areas by a masking operator are presented in Fig. 9 for the transmittancebased model and for the infrared-based model proposed in [7]. To enable the comparison, smoothing and robust parameters were tuned similarly, and a unique layer comprising all clouds was considered. Globally, estimations are visually coherent. One can nevertheless notice few differences, as for instance, in noncloudy regions. Without the use of a masking operator, the transmittance-based model tends to reveal no motion in clearsky areas, while the infrared-based model tends to provide a nonzero solution in those regions. This appears to be a natural consequence of the absence of observations in clear-sky areas for transmittance images, contrarily to infrared images. Finally, a visual inspection does not clearly allow us to state the best model.

Nevertheless, an important remark is that the color dynamics of images related to the infrared-based model is inverted in comparison to transmittance images. This means that, in the case of a divergent motion in hydrostatic-equilibrium conditions, on the contrary of transmittance-based model, the infrared-based model will underestimate the divergent sources or sinks. Indeed, according to (6), mass dispersion is compensated by a decrease of brightness along the motion trajectories. In addition, in the case of infrared imagery, a brightness decrease implicates an increase of altitude, which is in contra-

${ }^{1}$ We note that the EUMETSAT extraction procedure was not correctly tuned when the classification was extracted (June 2004). After comparison with cloud classifications obtained by other methods, it appeared that general cloud coverage was under evaluated. The high-level cloud coverage was also found to be underestimated in favor of medium-level clouds. These differences should not change radically the following evaluation, since a large majority of cloudy pixels are correctly assigned. Nevertheless, better classification products are likely to significantly enhance the performance of the method.
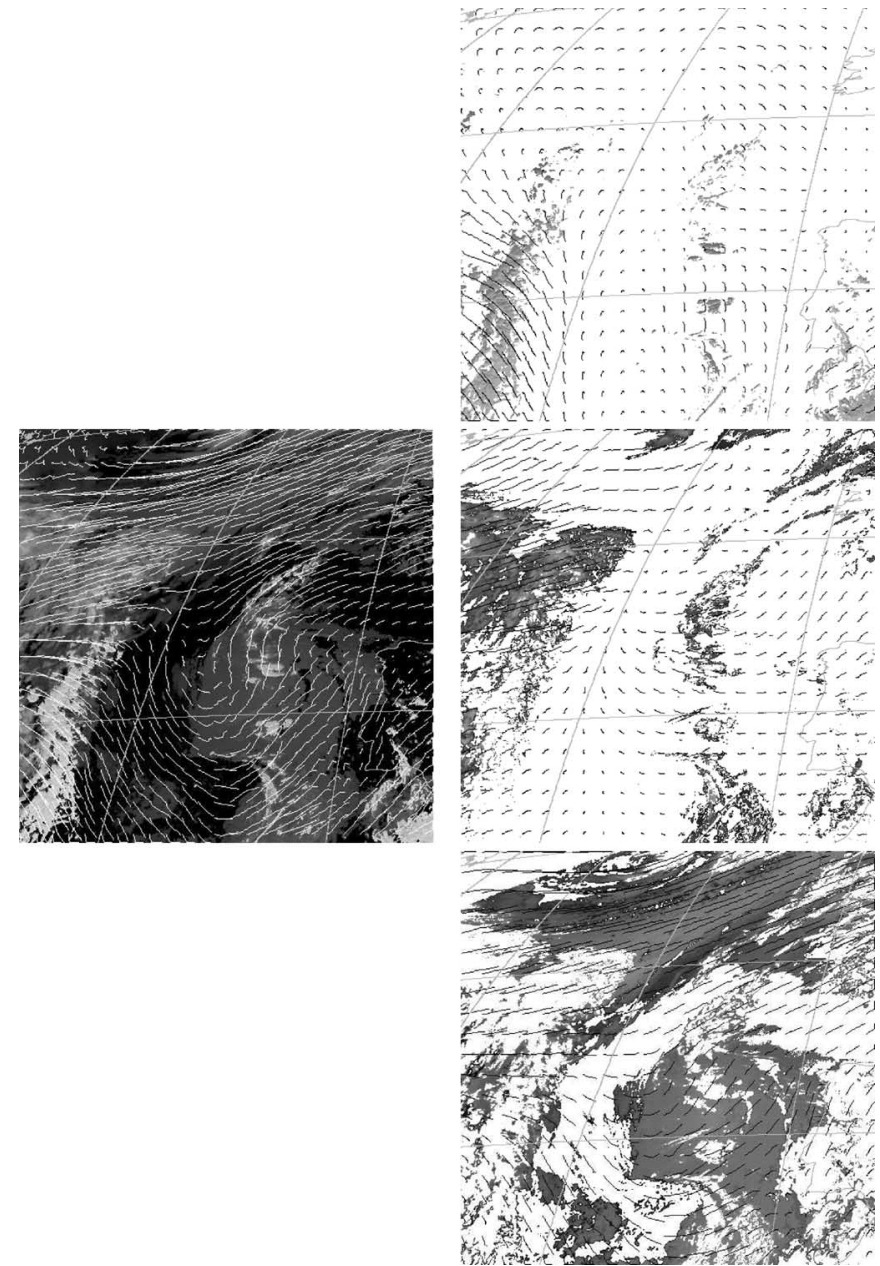

Fig. 10. Layer decomposition. On the left, trajectories estimated for a unique layer. On the right, trajectories related to the (top) higher, (middle) the intermediate, and (bottom) the lower layer. Gray lines represent coastal contours, meridians, and parallels (every $10^{\circ}$ ).

diction with altitude decrease induced by the mass dispersion. Moreover, the only model providing a physically sound scheme for layer decomposition is the transmittance-based one.

The global motion decomposition into a set of three independent-layer motions is presented in Fig. 10. Several trajectory differences are striking. Some of them are very relevant. For instance, near the middle of the image, the lower layer possesses a southward motion while the intermediate layer moves northward. This difference in motion is partially estimated with the layer decomposition. However, other trajectories related to the intermediate and higher layers seem to be inconsistent with the visual inspection of the image sequence, particularly for regions far from cloud boundaries.

Indeed, the multiresolution framework remains insufficient to catch large displacement of fine structures when important structures underneath move in an opposite direction. This is the case with the small clouds of the intermediate and high layers moving above a large stratus belonging to the lower layer. The problem is tackled using vector constraints. Let us briefly describe the correlation-based method, proposed in [37], which has been used to derive wind-vector constraints associated with the infrared meteorological images. In this 


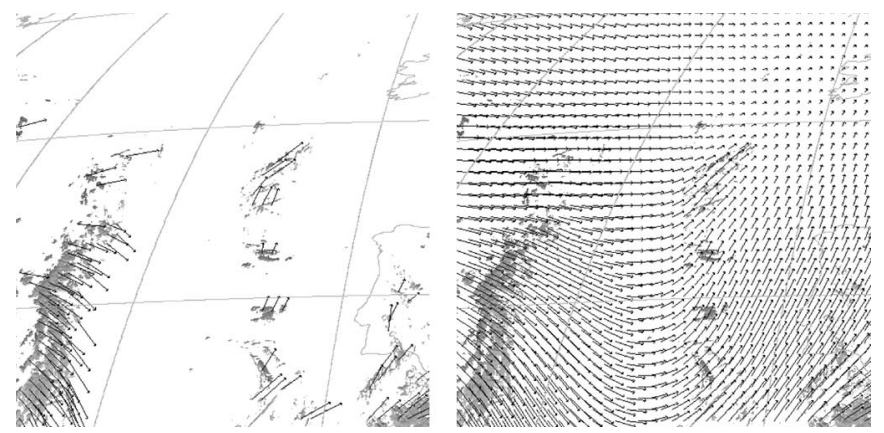

Fig. 11. Correlation constraints. Correlation-based sparse-wind-field vectors related to the highest layer. (Left) Interpolated using spatio-temporal smoothing in order to produce a dense-wind-field estimate relevant of large scales (right). Gray lines represent coastal contours, meridians, and parallels (every $10^{\circ}$ ).

algorithm, the vector-extraction procedure is done on a regular pixel grid and undergoes a series of quality tests: removal of outliers and spatio-temporally nonconsistent vectors. However, no height assignment or layer differentiation is undertaken in the method. In order to avoid the extraction of erroneous wind vectors, correlation-based vectors calculated on possibly occluded regions have been removed, and confidence factors $g^{i}$ were fixed to cross-correlational values associated with the vector constraints. An example of a sparse field of vector constraints related to the higher layer is displayed in Fig. 11. It also presents the large-scale dense-motion field, which has been produced by interpolating the sparse field in our collaborative scheme using spatio-temporal smoothing.

Spatio-temporal smoothing can remove ambiguities in those regions where no observations are available by propagating a solution in time and space. Fig. 12 shows the vorticity and divergence components of the large-scale wind field predicted by shallow-water large-eddy simulation. In particular, the introduction of such a priori temporal constraints enforces the large-scale wind-field solution to be consistent with previous estimates. This consistency is illustrated in Fig. 12.

Enhancements in motion estimation of the higher layer brought by the collaborative estimation scheme and by spatiotemporal smoothing are presented in Fig. 13. It can be noticed in this comparative figure that the collaborative scheme succeeds in characterizing large displacement of small cirrus while the multiresolution approach fails. Moreover, one can notice that the introduction of spatio-temporal constraints smooths trajectory discontinuities and propagates motion in regions where observations are missing. Improvements brought by spatiotemporal smoothing on trajectories related to the other layers are presented in Fig. 14. In particular, in the middle of the image, one can notice a better retrieval of the northward motion related to sparse clouds of the intermediate layer, which are above a stratus underneath moving southward.

\section{CONCLUSION}

In this paper, we have presented a new method for estimating winds in a stratified atmosphere from satellite image sequences of top-of-cloud pressure. The proposed motion-

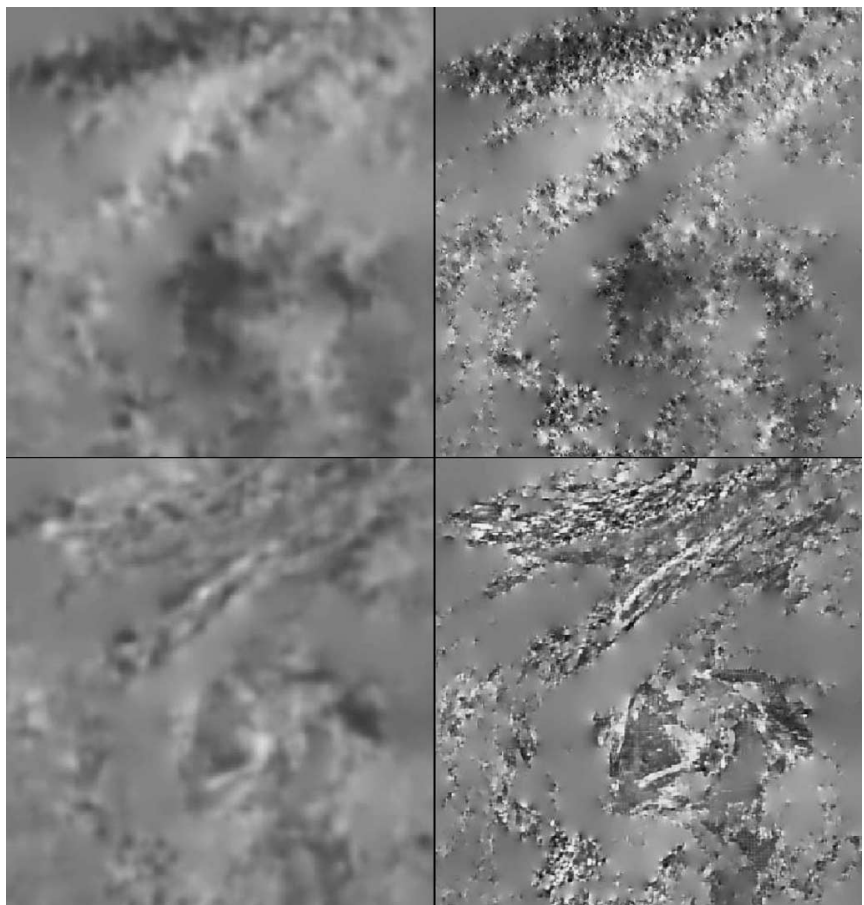

Fig. 12. Vorticity and divergence temporal constraints. On the left, lower layer vorticity (above) and divergence (below) predicted fields are introduced as large-scale spatio-temporal smoothers. Resulting large-scale vorticity (above) and divergence (below) estimates are displayed on the right. The image spatial domain corresponds here to the area above the North Atlantic Ocean selected previously.

estimation method is based on the minimization of a functional, including a two-part global smoother.

The data term relies on the ICE mass-conservation model. Indeed, the hydrostatic assumption enables a layer decomposition of the atmosphere. This decomposition is used to derive, relatively to each layer, transmittance observations from top-of-cloud pressure satellite images. Resulting observations verify independent mass-conservation models. To overcome the problem of sparse observations, a robust estimator is introduced in the data term.

A novel spatio-temporal smoother is proposed. An approximation of shallow-water momentum equations expressed in a divergence-vorticity form is used to derive temporal coherence constraints. These temporal constraints are combined with a robust second-order smoother preserving divergent and vorticity structures of the flow.

In order to capture mesoscale dynamics, a collaborative estimation scheme is developed. In a first stage, large displacements are estimated in a variational framework on the basis of correlation-based constraints and image observations, while in a second stage, the solution is refined using a motion-compensated function. This two-stage estimation scheme constitutes an advantageous alternative to the standard multiresolution framework.

On both synthetic images and real satellite images, the merit of the novel data model and of the introduction of correlationbased and temporal constraints have been demonstrated. A scalar image sequence generated by DNS of a turbulent 2-D flow was chosen for an exhaustive evaluation. Analyses were 

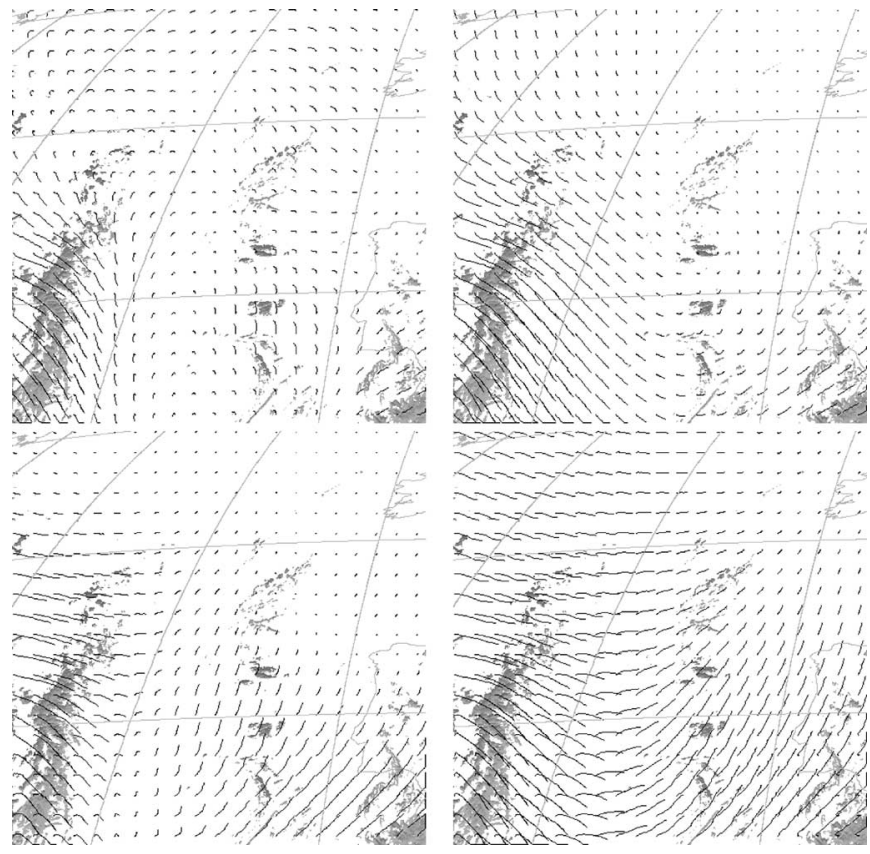

Fig. 13. Collaborative approach and spatio-temporal smoothing influence on the estimation of wind field for the higher layer. (Top) Trajectory reconstruction for multiresolution estimation scheme (left) without and (right) with spatiotemporal smoothing. (Bottom) Trajectory reconstruction for the two-stage collaborative estimation scheme (left) without and (right) with spatio-temporal smoothing. Gray lines represent coastal contours, meridians, and parallels (every $\left.10^{\circ}\right)$.

performed using this benchmark data in the image and in the spectral domain. In particular, the improvements brought by a two-stage estimation scheme with noisy correlation-based constraints and by the spatio-temporal smoother were quantified. By qualitative comparisons, the novelties of the method were also evaluated on a real meteorological image sequence. The two-stage estimation scheme, the proposed observation model, and propagation model proved their relevance for the characterization of mesoscale dynamics relatively to atmospheric layers.

In view of the various meteorological studies relying on the analysis of experimental data of atmospheric dynamics, we believe that the multilayer horizontal-wind-field estimation technique we have presented constitutes a valuable tool. The extension of the layer-independent transmittance-based model to a global model able to capture layer interactions via mass exchanges driven by vertical winds is our next step toward a more complete characterization of distribution of 3-D atmospheric winds.

\section{APPENDIX I \\ GAUSS-SEIDEL Solver FOR Motion Estimation}

For better and faster convergence, the minimization with respect to the displacement field $\mathbf{v}^{k}$ is performed using the coarse-to-fine multigrid technique introduced in [25]. In this optimization scheme, the searched displacement field is constrained to be piecewise parametric relative to an image partition that becomes increasingly finer according to a grid level. More precisely, at "grid" level $l$, the pixel grid is partitioned
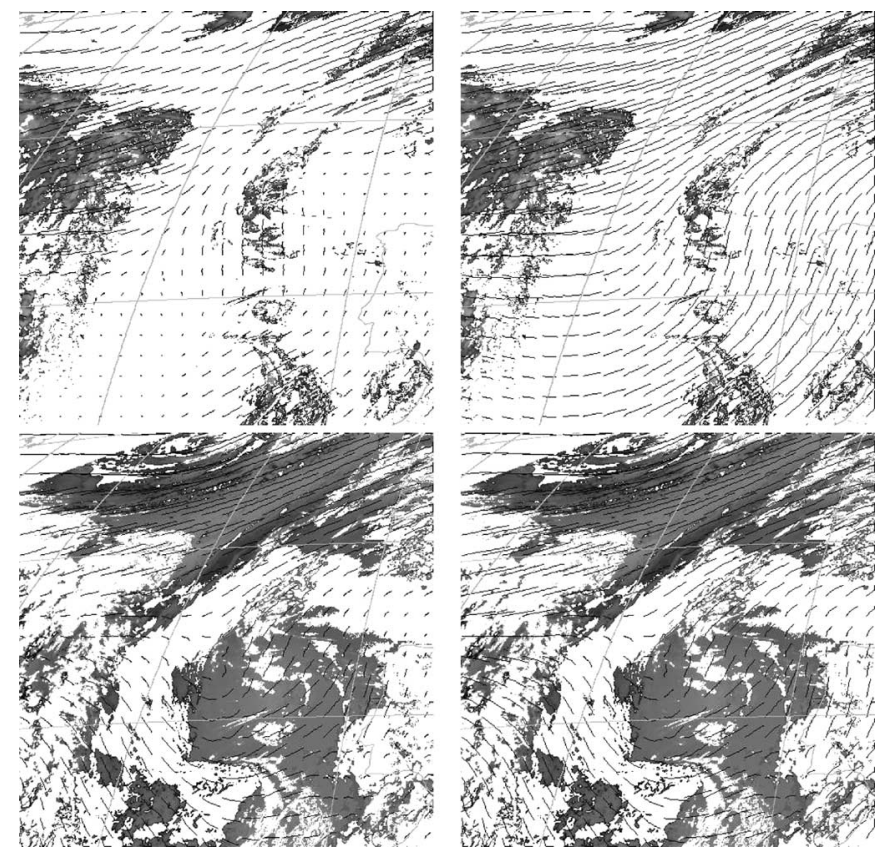

Fig. 14. Enhancements brought by spatio-temporal smoothing. Estimation of wind fields related to the middle and the lower layer using a collaborative estimation scheme (right) with or (left) without spatio-temporal smoothing. Reconstructed trajectories correspond to (bottom) the low and (top) to the medium layer motions. Gray lines represent coastal contours, meridians, and parallels (every $10^{\circ}$ ).

into $B_{l}^{n}$ blocks of size $2^{l} \times 2^{l}$, and the displacement field is constrained to satisfy

$$
\mathbf{v}^{k}(\mathbf{s})=P_{l}(\mathbf{s}) \theta_{l}^{n}+\overline{\mathbf{v}}^{k}(\mathbf{s}), \quad \forall n, \forall \mathbf{s} \in B_{l}^{n}
$$

where $\overline{\mathbf{v}}^{k}(\mathbf{s})$ is the current estimate and where $P_{l}(\mathbf{s})$ is a matrix that depends on the chosen parametrization at level $l$, and $\theta_{l}^{n}$ is the parameter vector for block $B_{l}^{n}$. Until grid level $l>2$, the affine parametrization is used, i.e.,

$$
\forall l, P_{l}(\mathbf{s}=(x, y))=\left[\begin{array}{llllll}
1 & x & y & 0 & 0 & 0 \\
0 & 0 & 0 & 1 & x & y
\end{array}\right] ; \quad \theta_{l}^{n} \in \mathbb{R}^{6}
$$

whereas for grid level $l \leq 2$, a constant parametric model is chosen, i.e.,

$$
\forall l, P_{l}(\mathbf{s}=(x, y))=\left[\begin{array}{ll}
1 & 0 \\
0 & 1
\end{array}\right] ; \quad \theta_{l}^{n} \in \mathbb{R}^{2} .
$$

At level $l$, (26) is rewritten with respect to the set of parameter vectors $\theta_{l}=\theta_{l}^{n}$ which defines $\mathbf{v}^{k}$ via (36). This symbolic rewriting is explained in [25]. It yields a quadratic-cost function, whose minimum corresponds to the solution of linear systems $A \theta_{l}^{n}=b$. In those systems, matrices $A$ and vectors $b$ are functions of the other variables used in the minimization. More details on the Gauss-Seidel resolution related to this cost term are provided in [7].

For the minimization of the energy defined in (26) with respect to $\overline{\mathbf{v}}^{k}$, matrix $A$ and vector $b$ have to be slightly modified because of the contribution of the new energy functions $\gamma J_{c}(\cdot)$ and $\beta J_{p}(\cdot)$ derived from (27) and (28). Thus, a new system 
is defined as $A^{\prime} \theta_{l}^{n}=b^{\prime}$. The new matrix $A^{\prime}$ is the sum of the previous matrix $A$ and of $A_{c}$ and $A_{p}$, which denote the contribution of the correlation-based and the propagated-field constraint, respectively. Similarly, the new vector $b^{\prime}$ is the sum of the previous vector $b$ and of $b_{c}$ and $b_{p}$, which denote the contribution of the correlation-based and the propagated-field constraint, respectively.

Let us denote the Kronecker product by $\otimes$ and the components of wind fields $\overline{\mathbf{v}}^{k}, \overline{\mathbf{v}}_{c}^{i}$ and $\overline{\mathbf{v}}_{p}^{k}$ by $(\bar{u}, \bar{v}),\left(\bar{u}_{p}, \bar{v}_{p}\right)$ and $\left(\bar{u}_{c}^{i}, \bar{v}_{c}^{i}\right)$. After a few analytical calculations, one can demonstrate that the previously introduced matrix and vectors are expressed as

$$
\begin{aligned}
A_{c} & =\gamma \sum_{i=1}^{K} g^{i} \mathcal{N}^{i}\left(\mathbf{s}^{i}-\mathbf{s}\right)\left[\begin{array}{ll}
1 & 0 \\
0 & 1
\end{array}\right] \otimes c \\
b_{c} & =\gamma \sum_{i=1}^{K} g^{i} \mathcal{N}^{i}\left(\mathbf{s}^{i}-\mathbf{s}\right)\left[\begin{array}{l}
d\left(\bar{u}_{c}^{i}-\bar{u}\right) \\
d\left(\bar{v}_{c}^{i}-\bar{v}\right)
\end{array}\right] \\
A_{p} & =\beta\left[\begin{array}{ll}
1 & 0 \\
0 & 1
\end{array}\right] \otimes c \\
b_{p} & =\beta\left[\begin{array}{l}
d\left(\bar{u}^{p}-\bar{u}\right) \\
d\left(\bar{v}^{p}-\bar{v}\right)
\end{array}\right]
\end{aligned}
$$

where, for the affine model, the coefficients read

$$
\begin{aligned}
c & =\left[\begin{array}{ccc}
1 & x & y \\
x & x^{2} & x y \\
y & x y & y^{2}
\end{array}\right] \\
d & =\left[\begin{array}{lll}
1 & x & y
\end{array}\right]^{\top}
\end{aligned}
$$

whereas, for the constant model, they are reduced to

$$
c=1 ; \quad d=1 .
$$

\section{APPENDIX II}

\section{Scale Analysis of NAVIER-STOKES Equations}

For mesoscale atmospheric motions observed through a METEOSAT images sequence, which has been low-pass filtered to obtain a characteristic horizontal scale of $100 \mathrm{~km}$, the following characteristic scales hold.

$\begin{array}{ll}U \sim 10 \mathrm{~m} \cdot \mathrm{s}^{-1} & \text { Horizontal velocity } \\ L \sim 10^{5} \mathrm{~m} & \text { Length } \\ H \sim 10^{3} \mathrm{~m} & \text { Depth } \\ \delta p \sim 10^{3} \mathrm{~Pa} & \text { Horizontal Pressure Fluctuation } \\ \rho_{0} \sim 1 \mathrm{~kg} \cdot \mathrm{m}^{-3} & \text { Density } \\ \Delta t \sim 10^{3} \mathrm{~s} & \text { Temporal Sampling Period } \\ \nu \sim 10^{-5} \mathrm{~m}^{2} \cdot \mathrm{s}^{-1} & \text { Friction Coefficient } \\ a \sim 10^{6} \mathrm{~m} & \text { Earth's Radius } \\ \Upsilon \sin \phi \sim 10^{-4} \mathrm{~s}^{-1} & \text { Coriolis Coefficient }\end{array}$

Scale analysis of terms in the horizontal momentum (29)

$$
\left\{\begin{array}{l}
\frac{d \bar{u}}{d t}-\frac{\bar{u} \bar{v} \tan \phi}{a}+\frac{\bar{p}_{x}}{\rho_{0}}=+2 \Upsilon \bar{v} \sin \phi+\mathcal{F}+\mathcal{T}_{\bar{u}} \\
\frac{d \bar{u}}{d t}-+\frac{\bar{u}^{2} \tan \phi}{a}+\frac{\bar{p}_{y}}{\rho_{0}}=-2 \Upsilon \bar{u} \sin \phi+\mathcal{F}+\mathcal{T}_{\bar{v}}
\end{array}\right.
$$

using the characteristic scales reads

\begin{tabular}{c|ccccc} 
& $\mathrm{A}$ & $\mathrm{B}$ & $\mathrm{C}$ & $\mathrm{D}$ & $\mathrm{E}$ \\
\hline$x-$ eq. & $\frac{d \bar{u}}{d t}$ & $2 \Upsilon \bar{v} \sin \phi$ & $\frac{\bar{u} \bar{v} \tan \phi}{a}$ & $\frac{1}{\rho} \frac{\partial \bar{p}}{\partial x}$ & $\mathcal{F}$ \\
$y$ - eq. & $\frac{d \bar{v}}{d t}$ & $2 \Upsilon \bar{u} \sin \phi$ & $\frac{\bar{u}^{2} \tan \phi}{a}$ & $\frac{1}{\rho} \frac{\partial \bar{p}}{\partial y}$ & $\mathcal{F}$ \\
scales & $\frac{U}{\Delta t}$ & $\Upsilon \sin \phi U$ & $\frac{U^{2}}{a}$ & $\frac{\delta p}{\rho L}$ & $\frac{\nu U}{H^{2}}$ \\
$\left(\mathrm{~m} \cdot \mathrm{s}^{-2}\right)$ & $10^{-2}$ & $10^{-3}$ & $10^{-4}$ & $10^{-2}$ & $10^{-10}$ \\
\hline
\end{tabular}

We conclude that terms $\mathrm{C}$ and $\mathrm{E}$ can be neglected in comparison to the other terms. Moreover, it is well known that, for high Reynolds numbers characteristic of atmospheric flows, due to the energy dissipation at unobservable scales, turbulent viscosity dissipation $\mathcal{T}$ cannot be neglected in Navier-Stokes numerical simulations [33], [35]. We obtain, thus, the momentum equations written in a vectorial form

$$
\rho_{0} \frac{d \overline{\mathbf{v}}}{d t}=-\nabla \bar{p}-2 \Upsilon \sin \phi\left[\begin{array}{cc}
0 & -1 \\
1 & 0
\end{array}\right] \overline{\mathbf{v}}+\mathcal{T} .
$$

\section{ACKNOWLEDGMENT}

The authors would like to thank the EUMETSAT consortium for providing and carefully preprocessing the data.

\section{REFERENCES}

[1] T. Bellerby, "Modeling and estimation of multiresolution stochastic processes," IEEE Trans. Geosci. Remote Sens., vol. 44, no. 12, pp. 3639 3648, Dec. 2006.

[2] D. Béréziat, I. Herlin, and L. Younes, "A generalized optical flow constraint and its physical interpretation," in Proc. Conf. Comput. Vis. Pattern Recog., Hilton Head Island, SC, 2000, vol. 2, pp. 487-492.

[3] J. Bergen, P. Burt, R. Hingorani, and S. Peleg, "A three-frame algorithm for estimating two-component image motion," IEEE Trans. Pattern Anal. Mach. Intell., vol. 14, no. 9, pp. 886-896, Sep. 1992.

[4] M. Black and P. Anandan, "The robust estimation of multiple motions: Parametric and piecewise-smooth flow fields," Comput. Vis. Image Underst., vol. 63, no. 1, pp. 75-104, Jan. 1996.

[5] A. Bruhn, J. Weickert, T. Kohlberger, and C. Schnoerr, "A multigrid platform for real-time motion computation with discontinuity-preserving variational methods," Int. J. Comput. Vis., vol. 70, no. 3, pp. 257-277, Dec. 2006.

[6] A. Chorin, "Numerical study of slightly viscous flow," J. Fluid Mech., vol. 57 , no. 4 , pp. 785-796, 1973.

[7] T. Corpetti, E. Mémin, and P. Pérez, "Dense estimation of fluid flows," IEEE Trans. Pattern Anal. Mach. Intell., vol. 24, no. 3, pp. 365-380, Mar. 2002.

[8] A. Cuzol and E. Memin, "Vortex and source particles for fluid motion estimation," in Proc. 5th Int. Conf. Scale-Space PDE Methods Comput. Vis., Hofgeismar, Germany, 2005, pp. 254-266.

[9] A. de Saint-Venant, "Theorie du mouvement non-permanent des eaux avec application aux crues des rivieres et l'introduction des marees dans leur lit," C. R. Acad. Sci. Paris, vol. 73, pp. 147-154, 1871.

[10] F. Desalmand, A. Szantai, and M. Desbois, "An attempt to retrieve low cloud motion winds over land in the African monsoon flow on METEOSAT pictures," Geophys. Res. Lett., vol. 26, no. 3, pp. 319-322, 1999.

[11] J. Fitzpatrick, "The existence of geometrical density-image transformations corresponding to object motion," Comput. Vis. Graph. Image Process., vol. 44, no. 2, pp. 155-174, Nov. 1988.

[12] U. Frisch, Turbulence: The Legacy of A. N. Kolmogorov. Cambridge, U.K.: Cambridge Univ. Press, 1995.

[13] P. Gamba, "Meteorological structures shape description and tracking by means of BI-RME matching," IEEE Trans. Geosci. Remote Sens., vol. 37, no. 2, pp. 1151-1161, Mar. 1999.

[14] D. Geman and G. Reynolds, "Constrained restoration and the recovery of discontinuities," Univ. Massachusetts Amherst, 1990. Tech. report.

[15] S. Geman and D. Geman, "Stochastic relaxation, Gibbs distributions and the Bayesian restoration of images," IEEE Trans. Pattern Anal. Mach. Intell., vol. PAMI-6, no. 6, pp. 721-741, Nov. 1984. 
[16] P. Holland and R. Welsch, "Robust regression using iteratively reweighted least-squares," Commun. Stat.-Theory Methods, vol. A6, no. 9, pp. 813$827,1977$.

[17] J. Holton, An Introduction to Dynamic Meteorology. New York: Academic, 1992

[18] B. Horn and B. Schunck, "Determining optical flow," Artif. Intell., vol. 17, no. 1-3, pp. 185-203, Aug. 1981.

[19] E. Huot, T. Isambert, I. Herlin, J. Berroir, and G. Korotaev, "Data assimilation of satellite images within an oceanographic circulation model," in Proc. IEEE Int. Conf. Acoust., Speech, Signal Process., Toulouse, France, May 2006.

[20] R. Larsen, K. Conradsen, and B. Ersboll, "Estimation of dense image flow fields in fluids," IEEE Trans. Geosci. Remote Sens., vol. 36, no. 1, pp. 256-264, Jan. 1998.

[21] J. Leese, C. Novack, and B. Clark, "An automated technique for obtaining cloud motion from geosynchronous satellite data using cross correlation," J. Appl. Meteorol., vol. 10, no. 1, pp. 118-132, Feb. 1971.

[22] B. Lucas and T. Kanade, "An iterative image registration technique with an application to stereo vision," in Proc. IJCAI, 1981, pp. 674-679.

[23] H. Lutzin "Cloud processing for METEOSAT second generation," Eur. Org. Exploitation Meteorol. Satell. (EUMETSAT), Darmstadt, Germany, 1999. Tech. report. [Online]. Available: http://www.eumetsat.de

[24] N. N. Mansour, J. H. Ferziger, and W. C. Reynolds, "Large-eddy simulation of a turbulent mixing layer," Thermosciences Div., Dept. Mech. Eng., Stanford Univ., Stanford, CA, Tech. Rep. TF-11, 1978.

[25] E. Mémin and P. Pérez, "Dense estimation and object-based segmentation of the optical flow with robust techniques," IEEE Trans. Image Process., vol. 7, no. 5, pp. 703-719, May 1998.

[26] E. Mémin and P. Pérez, "Fluid motion recovery by coupling dense and parametric vector fields," in Proc. ICCV, 1999, pp. 620-625.

[27] E. Mémin and P. Pérez, "Hierarchical estimation and segmentation of dense motion fields," Int. J. Comput. Vis., vol. 46, no. 2, pp. 129-155, Feb. 2002.

[28] W. Menzel, W. Smith, and T. Stewart, "Improved cloud motion wind vector and altitude assignment using VAS," J. Clim. Appl. Meteorol., vol. 22, no. 3, pp. 377-384, Mar. 1983.

[29] N. Papenberg, A. Bruhn, T. Brox, S. Didas, and J. Weickert, "Highly accurate optic flow computation with theoretically justified warping," Int. J. Comput. Vis., vol. 67, no. 2, pp. 141-158, 2006.

[30] A. Ottenbacher, M. Tomasini, K. Holmund, and J. Schmetz, "Low-level cloud motion winds from METEOSAT high-resolution visible imagery," Weather Forecast., vol. 12, no. 1, pp. 175-184, Mar. 1997.

[31] J. Ravnik, L. Skerget, and M. Hribersek, "The wavelet transform for BEM computational fluid dynamics," Eng. Anal. Bound. Elem., vol. 28, no. 11, pp. 1303-1314, Nov. 2004.

[32] P. Ruhnau, A. Stahl, and C. Schnoerr, "On-line variational estimation of dynamical fluid flows with physics-based spatio-temporal regularization," in Proc. 28th Symp. German Assoc. Pattern Recog., Berlin, Germany, Sep. 2006, pp. 444-454.

[33] P. Sagaut, "Introduction a la simulation des grandes echelles pour les ecoulements de fluide incompressible," in Collection: Mathematiques et Applications 30. Berlin, Germany: Springer-Verlag, 1998.

[34] J. Schmetz, K. Holmlund, J. Hoffman, B. Strauss, B. Mason, V. Gaertner, A. Koch, and L. V. D. Berg, "Operational cloud-motion winds from METEOSAT infrared images," J. Appl. Meteorol., vol. 32, no. 7, pp. 1206-1225, Jul. 1993.

[35] J. Smagorinsky, "General circulation experiments with the primitive equations," Mon. Weather Rev., vol. 91, no. 3, pp. 99-164, Mar. 1963.

[36] D. Suter, "Motion estimation and vector splines," in Proc. Conf. Comput. Vis. Pattern Recog., Seattle, WA, Jun. 1994, pp. 939-942.

[37] A. Szantai and F. Desalmand, "Using multiple channels from MSG to improve atmospheric motion wind selection and quality," in Proc. 7 th Int. Winds Workshop, EUMETSAT EUM P 42, Helsinki, Finland, Jun. 2004, pp. 307-314.

[38] G. Taylor, "The transport of vorticity and heat through fluids in turbulent motion," Proc. Lond. Math. Soc. Ser A, pp. 151-421, 1932.

[39] J. Weickert and C. Schnörr, "A theoretical framework for convex regularizers in PDE-based computation of image motion," Univ. Mannheim, Mannheim, Germany, Tech. Rep. 13/2000, 2000.

[40] J. Weickert and C. Schnörr, "Variational optic-flow computation with a spatio-temporal smoothness constraint," J. Math. Imaging Vis., vol. 14, no. 3, pp. 245-255, May 2001.

[41] G. S. Winckelmans, T. S. Lund, D. Carati, and A. A. Wray, "A priori testing of subgrid-scale models for the velocity pressure and vorticityvelocity formulations," in Proc. Turbulence Res., Summer Program, 1996, pp. $309-328$.
[42] J. Yuan, C. Schnoerr, and E. Memin, "Discrete orthogonal decomposition and variational fluid flow estimation," J. Math. Imaging Vis., vol. 28, no. 1, pp. 67-80, May 2007.

[43] L. Zhou, C. Kambhamettu, and D. Goldgof, "Fluid structure and motion analysis from multi-spectrum 2D cloud images sequences," in Proc. Conf. Comput. Vis. Pattern Recog., 2000, vol. 2, pp. 744-751.

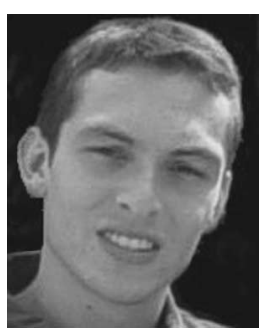

Patrick Héas received the Ph.D. degree in computer science and telecommunication from the National Aerospace Engineering School, Toulouse, France, in 2005 .

From 2001 to 2005, he worked in collaboration with the German Aerospace Center, the Computer Science Research Institute of Toulouse, and the French Space Agency. Since June 2005, he has been with the VISTA Group, Institut $\mathrm{Na}$ tional de Recherche en Informatique et en Automatique, Rennes, France. His research interests include spatio-temporal learning for dynamic scene understanding and variational methods for fluid-motion analysis.

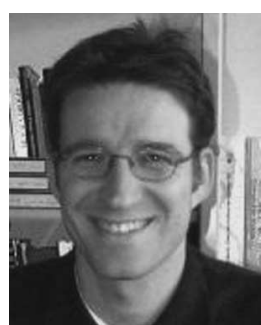

Etienne Mémin received the Ph.D. degree in computer science and the Habilitation degree from the University of Rennes I, Rennes, France, in 1993 and 2003, respectively.

From 1994 to 1999, he was an Assistant Professor in computer science with the University of South Brittany, Vannes, France. He is currently an Assistant Professor in computer science with the University of Rennes I. His major research interest includes motion analysis (motion estimation, motion segmentation, and tracking), energy-based modeling, and parallel processing. Since the last few years, he has been particularly interested in motion-analysis issues for image sequences depicting fluid phenomenon.

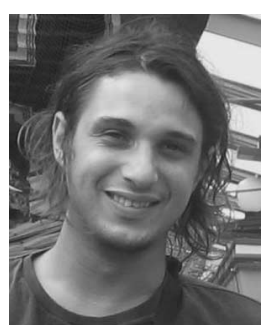

Nicolas Papadakis was born in 1981. He received the degree in applied mathematics from the $\mathrm{Na}$ tional Institute of Applied Sciences, Rouen, France, in 2004. Since 2004, he has been working toward the Ph.D. degree in applied mathematics from the University of Rennes, Rennes, France.

His main research interests are in variational models for tracking in image sequences.

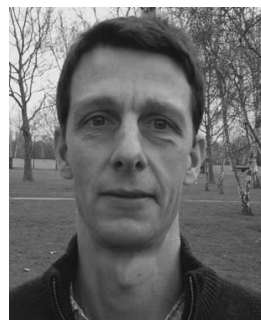

André Szantai received the Ph.D. degree specializing in the analysis of cloud and atmospheric motions on geostationary satellite IMAGESAT from the Université de Paris VI, Paris, France, in 1996.

$\mathrm{He}$ has been a permanent Engineer with the Centre National de la Recherche Scientifique/Laboratoire de Météorologie Dynamique, Ecole Polytechnique, Palaiseau, France, since 1985. He has published several papers on the subject of the analysis of cloud and atmospheric motions on geostationary satellite IMAGESAT and participates regularly in international workshops where all the main meteorological and space institutions working on the subject meet. 